The AstrophysicAL JouRNAL, 547:872-884, 2001 February 1

(C) 2001. The American Astronomical Society. All rights reserved. Printed in U.S.A.

\title{
INTERSTELLAR EXTINCTION AND POLARIZATION IN THE TAURUS DARK CLOUDS: THE OPTICAL PROPERTIES OF DUST NEAR THE DIFFUSE/DENSE CLOUD INTERFACE
}

\author{
D. C. B. Whittet, ${ }^{1}$ P. A. Gerakines, ${ }^{1,2}$ J. H. Hough, ${ }^{3}$ AND S. S. Shenoy ${ }^{1}$ \\ Received 2000 June 29; accepted 2000 September 27
}

\begin{abstract}
Observations of interstellar linear polarization in the spectral range $0.35-2.2 \mu \mathrm{m}$ are presented for several stars reddened by dust in the Taurus region. Combined with a previously published study by Whittet et al., these results represent the most comprehensive data set available on the spectral dependence of interstellar polarization in this nearby dark cloud (a total of 27 sight lines). Extinction data for these and other reddened stars in Taurus are assembled for the same spectral range, combining published photometry and spectral classifications with photometry from the Two Micron All Sky Survey. The polarization and extinction curves are characterized in terms of the parameters $\lambda_{\max }$ (the wavelength of maximum polarization) and $R_{V}$ (the ratio of total to selective extinction), respectively. The data are used to investigate in detail the question of whether the optical properties of the dust change systematically as a function of environment, considering stars observed through progressively more opaque (and thus progressively denser) regions of the cloud. At low visual extinctions $\left(0<A_{V}<3\right.$ ), the dust has $R_{V}=2.97 \pm 0.15$, implying optical properties closely similar to those of "normal" dust in the diffuse interstellar medium (ISM). However, $\lambda_{\max }$ is significantly higher than the diffuse-ISM average toward some stars in this extinction range, a result which we attribute to size-dependent failure of the grain alignment mechanism. For extinctions $A_{V}>3$, real changes in grain properties occur, characterized by observed $R_{V}$ values in the range 3.5-4.0. A simple model for the development of $R_{V}$ with $A_{V}$ suggests that $R_{V}$ may approach values of 4.5 or more in the densest regions of the cloud. The transition between "normal" extinction and "dense cloud" extinction occurs at $A_{V} \sim 3.2$, a value coincident with the threshold extinction above which $\mathrm{H}_{2} \mathrm{O}$-ice is detected on grains within the cloud. Changes in $R_{V}$ are thus either a direct consequence of mantle growth or occur under closely similar physical conditions. Dust in Taurus appears to be in a different evolutionary state compared with other nearby dark clouds, such as $\rho \mathrm{Oph}$, in which coagulation is the dominant physical process.
\end{abstract}

Subject headings: dust, extinction - ISM: individual (Taurus Dark Cloud) - ISM: magnetic fields polarization

\section{INTRODUCTION}

The extensive complex of dark clouds in the Taurus region (hereafter TDC) provides an excellent opportunity to study the properties of interstellar matter under physical conditions close to the interface between diffuse atomic and dense molecular phases. At a distance of 140 pc (Elias 1978a; Kenyon, Dobryzycka, \& Hartmann 1994), the TDC is one of the closest regions of current star formation to the solar system. Little foreground or background dust appears to be present, virtually all of the extinction and reddening of objects within and behind the cloud occurring in the cloud itself (Straižys \& Meištas 1980; Meištas \& Straižys 1981). Studies of the $3 \mu \mathrm{m} \mathrm{H}_{2} \mathrm{O}$-ice absorption feature and its correlation with visual extinction have shown that icy grain material is present only at extinctions above some threshold; i.e., the dust grains acquire detectable ice mantles at $A_{V}>A_{\mathrm{th}}$, where $A_{\mathrm{th}} \approx 3$ is the threshold extinction in magnitudes (Whittet et al. 1983; 1988; Smith, Sellgren \& Brooke 1993; Teixeira \& Emerson 1999; Murakawa, Tamura, \&

\footnotetext{
${ }^{1}$ Department of Physics, Applied Physics \& Astronomy, Rensselaer Polytechnic Institute, Troy, NY 12180.

${ }_{2}$ Present address: NASA/Goddard Space Flight Center, Astrochemistry Branch (Code 691), Greenbelt, MD 20771.

${ }^{3}$ Department of Physical Sciences, University of Hertfordshire, College Lane, Hatfield, Hertfordshire, AL10 9AB, UK.
}

Nagata 2000). The value of $A_{\mathrm{th}}$ varies from cloud to cloud, and is generally lower in the TDC compared with other dark clouds studied to date (e.g., Whittet et al. 1996 and references therein), consistent with an absence of internal sources of desorbing radiation (Williams, Hartquist, \& Whittet 1992). In this paper, we take the threshold extinction to be a marker of the transition between the diffuse outer layers of the cloud and the cold, dense interior. It is likely that the ice threshold is quite closely related to the phase change between predominantly atomic and predominantly molecular gas. Ice mantles are predicted to form primarily by surface reactions between accreted $\mathrm{H}$ and $\mathrm{O}$ atoms (Jones \& Williams 1984), and thus grow most efficiently on grains immersed in the densest gas that has a significant atomic $\mathrm{H}$ content, i.e., close to the $\mathrm{H} \mathrm{I} / \mathrm{H}_{2}$ interface. Note also that millimeter-wave emission from gas phase $\mathrm{CO}$, the primary observational tracer of $\mathrm{H}_{2}$, is likewise detected only above a threshold extinction that is quantitatively similar to the ice threshold in the TDC (Frerking, Langer, \& Wilson 1982).

Several physical processes can lead to changes in the optical (extinction and polarization) properties of dust in dense regions of dark clouds. Mantle growth, in which atoms and molecules accrete from the gas to form icy coatings, has already been mentioned. The rate of growth is expected to be independent of the size of the particle in the absence of desorbing radiation, and the appearance of 
mantles should thus introduce a systematic shift of the entire size distribution toward larger sizes. Grains may also grow by grain-grain collisions that lead to coagulation, and this will tend to redistribute the size distribution as small grains become depleted by inclusion in larger ones. It is the latter process that is thought to be primarily responsible for systematic changes observed in the extinction curve (e.g., Cardelli, Clayton, \& Mathis 1989; Mathis 1996). Finally, when polarization is considered, variations in the efficiency of alignment by the ambient magnetic field must also be taken into account. If these changes are size-dependent, they will affect the spectral dependence as well as the degree of polarization.

Whereas the distribution of reddening and polarizing material in the TDC has been studied quite extensively (e.g., Straižys \& Meištas 1980; Meištas \& Straižys 1981; Moneti et al. 1984; Arce \& Goodman 1999), the wavelengthdependence of these phenomena has been investigated only for a relatively few lines of sight. Available data for stars with low to moderate extinctions (Vrba \& Rydgren 1985; Whittet et al. 1992; Kenyon et al. 1994) hint that the dust has generally "normal" optical properties, characterized by values of the parameters $R_{V}=A_{V} / E_{B-V}$ (the ratio of total to selective extinction) and $\lambda_{\max }$ (the wavelength of maximum linear polarization) close to mean values for the general interstellar medium (ISM), i.e., $R_{V} \approx 3.1$ and $\lambda_{\max } \approx$ $0.55 \mu \mathrm{m}$. However, objects with deep ice absorption features are generally not accessible to observation in the visible because of their high extinctions. The best studied case of a visible star with detectable ice absorption is HD 29647: its extinction curve has a somewhat elevated value of $R_{V}$ ( 3.6; Crutcher 1985; Vrba \& Rydgren 1985; Straižys, Cernis \& Hayes 1985), and displays more spectacular departures from average behavior in the ultraviolet (Cardelli \& Savage 1988).

The main aim of this paper is a detailed study of the wavelength dependences of interstellar polarization and extinction in the TDC, based on a greatly extended sample of sight lines compared with previous work. In particular, we focus on the question of whether the optical properties of the dust change systematically as a function of environment near the ice threshold. Our data set, described in $\S 2$, includes visible and infrared broadband polarimetry, obtained with the United Kingdom Infrared Telescope, and infrared photometry extracted from the Two Micron All Sky Survey. The data are analyzed and discussed in $\S 3$. A simple model for the dependence of $R_{V}$ on visual extinction is described and compared with observations in $\S 4$. Constraints on the relative importance of mantle growth and coagulation as physical processes leading to changes in $R_{V}$ are discussed in $\S 5$. A summary of the principal results and suggestions for further research appear in the final section.

\section{THE DATA SET}

A total of 54 reddened stars in the general direction of the TDC complex are included in this study. The primary objectives of the observing program were (1) to extend the existing polarimetric data set for Taurus stars reported by Whittet et al. (1992) and (2) to determine reliable extinction parameters for stars with polarimetric data available. The final sample contains 27 targets with both polarization and extinction information. Most of our targets were selected from lists of visually bright $(V<12 \mathrm{mag})$ reddened and/or polarized stars (e.g., Straižys \& Meištas 1980; Moneti et al.
1984). ${ }^{4}$ In addition to visually-selected targets, we include three objects from the Elias (1978a) near infrared survey of the TDC. To increase the sample size for extinction studies, we also present a compilation of extinction data for a further 27 stars that lack spectropolarimetric data, selected from Vrba \& Rydgren (1985), Kenyon et al. (1994), and Arce \& Goodman (1999). ${ }^{5}$ All objects included, both in the polarimetry observing program and in the additional set of reddened stars, are thought to be field stars, i.e., situated behind the TDC complex and reddened by foreground dust within it. Embedded young stellar objects are specifically excluded, owing to uncertainties in their intrinsic colors and the possibility of circumstellar polarization or infrared emission.

\subsection{Polarimetry}

New observations of interstellar linear polarization were made for a total of 11 program stars during an observing run with the United Kingdom Infrared Telescope (UKIRT) at Mauna Kea Observatory, Hawaii, on the nights of 1995 January 6-8. The instrument used was the Hatfield polarimeter (Hatpol), described in detail by Hough, Peacock, \& Bailey (1991). Simultaneous observations were possible in six broadband filters $(U, B, V, R, I$, and one selected from $J$, $H, K)$. The effective wavelengths of the filters were $0.36,0.43$, $0.55,0.63,0.78,1.25,1.65$ and $2.20 \mu \mathrm{m}$, respectively. The data collection and reduction procedures were closely similar to those described in our previously published studies of interstellar polarization using this instrument (Whittet et al. 1992, 1994). Resulting values of the degree $\left(p_{\lambda}\right)$ and position angle $\left(\theta_{\lambda}\right)$ of polarization, and their associated uncertainties, are presented in Table 1 .

It is well known that the wavelength dependence of linear polarization can be described to a close approximation by the empirical formula known as the Serkowski law:

$$
p_{\lambda} / p_{\max }=\exp \left[-K \ln ^{2}\left(\lambda_{\max } / \lambda\right)\right]
$$

where $p_{\max }$ is the peak percentage polarization and $\lambda_{\max }$ the wavelength corresponding to $p_{\max }$ (Serkowski 1973; Serkowski, Mathewson \& Ford 1975). With $K$ treated as a free parameter, following Wilking et al. (1980) and Wilking, Lebofsky, \& Rieke (1982), least-squares fits of equation (1) to data for stars with a range of $\lambda_{\max }$ show that $K$ and $\lambda_{\max }$ are linearly correlated:

$$
K=c_{1} \lambda_{\max }+c_{2} .
$$

For a variety of interstellar environments and physical conditions, the average values of the slope and intercept are $c_{1}=1.66 \pm 0.09$ and $c_{2}=0.01 \pm 0.05$ (Whittet et al. 1992).

\footnotetext{
${ }^{4}$ Visually-selected objects are designated by their Henry Draper (HD) numbers throughout this paper. HD numbers for Taurus stars in the main Henry Draper Catalog have five digits, those in the Henry Draper Extension have six digits. Some authors, notably Kenyon et al. (1994), use SAO numbers in preference to HD numbers: the correspondence may be easily found, e.g., with reference to the Simbad database.

${ }^{5}$ Arce \& Goodman (1999) present reddening data for some 100 stars in the direction of the TDC. However, the vast majority have relatively low extinction $\left(A_{V}<3\right)$, and their spectral classifications do not include luminosity classes; hence the uncertainties in the color excesses and in $A_{V}$ and $R_{V}$ are larger than for typical stars in our sample. We selected for analysis the only stars (091085 and 141134) in the Arce \& Goodman compilation that have extinctions substantially above the ice threshold. Because of their relatively high extinctions, errors introduced by uncertainties in spectral types are less important.
} 
TABLE 1

ResUlts of BROADBAND POlaRimetry

\begin{tabular}{|c|c|c|c|}
\hline Star & Filter & $\begin{array}{l}p_{\lambda} \\
(\%)\end{array}$ & $\begin{array}{c}\theta_{\lambda} \\
(\mathrm{deg})\end{array}$ \\
\hline \multirow[t]{8}{*}{ HD $28225 \ldots \ldots}$. & $U$ & $1.53 \pm 0.05$ & $13.6 \pm 0.8$ \\
\hline & $B$ & $1.68 \pm 0.03$ & $12.5 \pm 0.5$ \\
\hline & $V$ & $1.93+0.03$ & $12.4+0.4$ \\
\hline & $R$ & $1.78 \pm 0.06$ & $13.0 \pm 0.9$ \\
\hline & $I$ & $1.58 \pm 0.06$ & $12.0 \pm 1.0$ \\
\hline & $J$ & $0.98 \pm 0.04$ & $12.9 \pm 1.4$ \\
\hline & $H$ & $0.54 \pm 0.03$ & $15.8 \pm 1.7$ \\
\hline & $K$ & $0.34 \pm 0.02$ & $10.9 \pm 1.5$ \\
\hline \multirow[t]{8}{*}{ HD $283367 \ldots \ldots$} & $U$ & $0.81 \pm 0.03$ & $133.4 \pm 1.2$ \\
\hline & $B$ & $1.05 \pm 0.04$ & $127.8 \pm 1.2$ \\
\hline & $V$ & $1.35 \pm 0.03$ & $126.8 \pm 0.7$ \\
\hline & $R$ & $1.41 \pm 0.04$ & $127.7 \pm 0.8$ \\
\hline & $I$ & $1.36 \pm 0.04$ & $124.3 \pm 0.8$ \\
\hline & $J$ & $0.97 \pm 0.05$ & $119.5 \pm 1.6$ \\
\hline & $H$ & $0.57+0.03$ & $122.6+2.3$ \\
\hline & $K$ & $0.36+0.04$ & $115.8+3.5$ \\
\hline \multirow[t]{8}{*}{ HD $283642 \ldots \ldots$} & $U$ & $1.59 \pm 0.08$ & $36.5 \pm 1.3$ \\
\hline & $B$ & $1.71 \pm 0.02$ & $34.2 \pm 0.4$ \\
\hline & V & $1.98 \pm 0.02$ & $32.1 \pm 0.3$ \\
\hline & $R$ & $1.97 \pm 0.02$ & $32.0 \pm 0.3$ \\
\hline & $I$ & $1.82 \pm 0.03$ & $29.0 \pm 0.5$ \\
\hline & $J$ & $1.14 \pm 0.02$ & $28.0 \pm 0.7$ \\
\hline & $H$ & $0.74 \pm 0.05$ & $26.1 \pm 1.7$ \\
\hline & $K$ & $0.42 \pm 0.03$ & $28.4 \pm 2.5$ \\
\hline \multirow[t]{8}{*}{ HD $283643 \ldots . .}$. & $U$ & $1.12 \pm 0.06$ & $33.1 \pm 1.4$ \\
\hline & $B$ & $1.16 \pm 0.03$ & $29.5 \pm 0.7$ \\
\hline & $V$ & $1.31+0.03$ & $24.7+0.7$ \\
\hline & $R$ & $1.30 \pm 0.03$ & $25.5 \pm 0.6$ \\
\hline & $I$ & $1.40 \pm 0.04$ & $21.8 \pm 0.8$ \\
\hline & $J$ & $0.81 \pm 0.03$ & $18.8 \pm 1.2$ \\
\hline & $H$ & $0.55 \pm 0.04$ & $18.9 \pm 2.1$ \\
\hline & $K$ & $0.28 \pm 0.06$ & $(12 \pm 7)$ \\
\hline \multirow[t]{8}{*}{ HD $283757 \ldots \ldots$} & $U$ & $2.35 \pm 0.05$ & $44.2 \pm 0.7$ \\
\hline & $B$ & $2.46 \pm 0.03$ & $41.7 \pm 0.3$ \\
\hline & $V$ & $2.95 \pm 0.02$ & $40.2 \pm 0.2$ \\
\hline & $R$ & $2.77 \pm 0.02$ & $40.0 \pm 0.2$ \\
\hline & $I$ & $2.73 \pm 0.03$ & $40.2 \pm 0.3$ \\
\hline & $J$ & $1.56 \pm 0.04$ & $37.0 \pm 1.0$ \\
\hline & $H$ & $0.97+0.05$ & $39.8+1.4$ \\
\hline & $K$ & $0.67 \pm 0.09$ & $38.7 \pm 3.5$ \\
\hline \multirow{8}{*}{ HD $283815 \ldots \ldots$} & $U$ & $2.40 \pm 0.06$ & $45.3 \pm 0.7$ \\
\hline & $B$ & $2.47 \pm 0.02$ & $45.9 \pm 0.3$ \\
\hline & $V$ & $2.82 \pm 0.03$ & $45.2 \pm 0.3$ \\
\hline & $R$ & $2.78 \pm 0.03$ & $44.9 \pm 0.3$ \\
\hline & $I$ & $2.58 \pm 0.03$ & $45.7 \pm 0.4$ \\
\hline & $J$ & $1.49 \pm 0.03$ & $46.1 \pm 0.8$ \\
\hline & $H$ & $0.92 \pm 0.04$ & $49.0 \pm 1.8$ \\
\hline & $K$ & $0.57 \pm 0.04$ & $44.2 \pm 2.5$ \\
\hline \multirow[t]{8}{*}{ HD $283877 \ldots . .}$. & $U$ & $1.23 \pm 0.03$ & $29.6 \pm 0.7$ \\
\hline & $B$ & $1.33 \pm 0.03$ & $28.1 \pm 0.6$ \\
\hline & $V$ & $1.68+0.04$ & $27.2+0.6$ \\
\hline & $R$ & $1.59 \pm 0.03$ & $25.7 \pm 0.5$ \\
\hline & $I$ & $1.53 \pm 0.02$ & $28.2 \pm 0.5$ \\
\hline & $J$ & $1.00 \pm 0.04$ & $25.3 \pm 1.4$ \\
\hline & $H$ & $0.67 \pm 0.06$ & $(7 \pm 5)$ \\
\hline & $K$ & $0.36 \pm 0.12$ & $(14 \pm 7)$ \\
\hline \multirow[t]{8}{*}{ HD $283879 \ldots \ldots$} & $U$ & $3.12 \pm 0.08$ & $33.9 \pm 0.8$ \\
\hline & $B$ & $3.43 \pm 0.04$ & $31.5 \pm 0.3$ \\
\hline & $V$ & $4.16 \pm 0.03$ & $29.6 \pm 0.2$ \\
\hline & $R$ & $4.07 \pm 0.03$ & $29.0 \pm 0.2$ \\
\hline & $I$ & $3.95 \pm 0.04$ & $29.1 \pm 0.2$ \\
\hline & $J$ & $2.36 \pm 0.04$ & $27.3 \pm 0.5$ \\
\hline & $H$ & $1.39 \pm 0.03$ & $27.8 \pm 0.7$ \\
\hline & $K$ & $0.86 \pm 0.05$ & $28.4 \pm 1.7$ \\
\hline
\end{tabular}

TABLE $1-$ Continued

\begin{tabular}{cccr}
\hline \hline Star & Filter & $\begin{array}{c}p_{\lambda} \\
(\%)\end{array}$ & \multicolumn{1}{c}{$\begin{array}{c}\theta_{\lambda} \\
(\mathrm{deg})\end{array}$} \\
\hline Elias 3...... & $V$ & $2.37 \pm 0.40$ & $107.6 \pm 4.7$ \\
& $R$ & $2.45 \pm 0.13$ & $107.1 \pm 1.5$ \\
& $I$ & $2.35 \pm 0.08$ & $108.6 \pm 0.9$ \\
& $J$ & $1.72 \pm 0.04$ & $108.7 \pm 0.7$ \\
& $H$ & $1.09 \pm 0.04$ & $109.6 \pm 1.0$ \\
Elias 29..... & $K$ & $0.65 \pm 0.02$ & $108.4 \pm 0.7$ \\
& $U$ & $3.2 \pm 1.3$ & $(47 \pm 13)$ \\
& $B$ & $4.22 \pm 0.16$ & $31.0 \pm 1.1$ \\
& $V$ & $4.37 \pm 0.07$ & $32.2 \pm 0.4$ \\
& $R$ & $4.39 \pm 0.04$ & $31.3 \pm 0.3$ \\
& $I$ & $3.91 \pm 0.06$ & $30.4 \pm 0.3$ \\
& $J$ & $2.37 \pm 0.02$ & $29.8 \pm 0.3$ \\
& $H$ & $1.49 \pm 0.03$ & $29.6 \pm 0.7$ \\
& $B$ & $4.46 \pm 0.36$ & $36.8 \pm 2.1$ \\
& $V$ & $5.57 \pm 0.07$ & $35.3 \pm 0.3$ \\
& $R$ & $5.31 \pm 0.05$ & $34.0 \pm 0.2$ \\
& $I$ & $4.94 \pm 0.04$ & $33.9 \pm 0.2$ \\
& $J$ & $2.86 \pm 0.06$ & $32.4 \pm 0.7$ \\
& $H$ & $1.81 \pm 0.05$ & $33.1 \pm 0.9$ \\
& $K$ & $1.14 \pm 0.04$ & $32.1 \pm 1.3$ \\
\hline
\end{tabular}

The dependence of $K$ on $\lambda_{\max }$, known as the Wilking law, implies a systematic decrease in the width of the polarization curve (proportional to $1 / K$ ) with increasing $\lambda_{\max }$. The simplest explanation of this behavior is a systematic reduction in the relative numbers of small, aligned grains in regions of high $\lambda_{\max }$ (Clayton et al. 1995; Martin, Clayton, \& Wolff 1999).

Fits to the observed values of $p_{\lambda}$ were calculated, using equation (1) with $K$ treated as a free parameter. The values of $p_{\max }, \lambda_{\max }$, and $K$ generated by the fits are listed in Table 2 . The fitting process was applied to all stars that satisfy our selection criteria and with Hatpol data available, either from Table 1 of this paper or from our previously published results (Whittet et al. 1992). The fit parameters given in Table 2 are generally consistent with those listed in Whittet et al. (1992) for stars in common (Elias 29 is an exception: the previous observations of this star are of much lower quality than those presented here). Also given in Table 2 are the mean, $\langle\theta\rangle$, and standard deviation, $\sigma(\theta)$, of the position angle for each star. Values of $\theta_{\lambda}$ with large uncertainty (in parentheses in Table 1) were excluded from the calculation. A few stars show evidence for position-angle rotation with wavelength, i.e., they exhibit a systematic trend in $\theta_{\lambda}$ with passband, and thus have values of $\sigma(\theta)$ appreciably greater than the mean observational error. We take $\sigma(\theta)>3^{\circ}$ as indicative of significant rotation: five stars (HD 29647, $283367,283642,283643$, and 283809) fall into this group.

As a consistency check, we plot the correlation of $\lambda_{\max }$ with $K$ (Table 2) in Figure 1. A linear least-squares fit to the 27 data points (shown as the solid line in Fig. 1) has slope and intercept $c_{1}=1.5 \pm 0.4$ and $c_{2}=0.1 \pm 0.1$ (eq. [2]). We conclude that the correlation for Taurus is not significantly different from the average relationship adopted by Whittet et al. (1992), shown as the dashed line in Figure 1.

\subsection{Infrared Photometry}

Previous studies of interstellar extinction in the TDC were hindered by a sparsity of infrared data needed to determine $R_{V}$ and $A_{V}(\S 2.3)$. This problem is now alleviated by the availability of results from the Two Micron All Sky 
TABLE 2

Serkowski Fit Parameters and Mean Position Angles

\begin{tabular}{|c|c|c|c|c|c|c|}
\hline Star & $\begin{array}{l}p_{\max } \\
(\%)\end{array}$ & $\begin{array}{c}\lambda_{\max } \\
(\mu \mathrm{m})\end{array}$ & $K$ & $\begin{array}{c}\langle\theta\rangle \\
(\mathrm{deg})\end{array}$ & $\begin{array}{c}\sigma(\theta) \\
(\operatorname{deg})\end{array}$ & References \\
\hline HD $28170 \ldots \ldots$. & $1.92 \pm 0.03$ & $0.55 \pm 0.01$ & $0.98 \pm 0.06$ & 89.4 & 0.7 & 1 \\
\hline HD $28225 \ldots \ldots \ldots$ & $1.88 \pm 0.04$ & $0.58 \pm 0.02$ & $1.05 \pm 0.09$ & 12.9 & 1.3 & 2 \\
\hline HD $28975 \ldots \ldots .$. & $3.17 \pm 0.03$ & $0.54 \pm 0.01$ & $0.89 \pm 0.05$ & 57.6 & 2.3 & 1 \\
\hline HD $29333 \ldots \ldots .$. & $5.25 \pm 0.07$ & $0.54 \pm 0.02$ & $0.89 \pm 0.07$ & 70.9 & 1.5 & 1 \\
\hline HD $29647 \ldots \ldots .$. & $2.30 \pm 0.02$ & $0.73 \pm 0.01$ & $1.15 \pm 0.04$ & 71.4 & 7.3 & 1 \\
\hline HD $29835 \ldots \ldots \ldots$ & $4.07 \pm 0.07$ & $0.50 \pm 0.03$ & $0.93 \pm 0.11$ & 23.0 & 1.5 & 1 \\
\hline HD $30168 \ldots \ldots .$. & $4.07 \pm 0.09$ & $0.53 \pm 0.03$ & $0.83 \pm 0.09$ & 24.6 & 0.9 & 1 \\
\hline HD $30675 \ldots \ldots .$. & $3.90 \pm 0.03$ & $0.52 \pm 0.01$ & $0.99 \pm 0.05$ & 58.7 & 1.3 & 1 \\
\hline HD $279652 \ldots \ldots$ & $1.29 \pm 0.09$ & $0.47 \pm 0.09$ & $0.78 \pm 0.26$ & 89.7 & 2.3 & 1 \\
\hline HD $279658 \ldots \ldots$. & $2.81 \pm 0.03$ & $0.53 \pm 0.01$ & $0.99 \pm 0.06$ & 146.0 & 1.4 & 1 \\
\hline HD $283367 \ldots \ldots$ & $1.43 \pm 0.05$ & $0.71 \pm 0.02$ & $1.23 \pm 0.11$ & 124.7 & 5.1 & 2 \\
\hline HD $283637 \ldots \ldots$ & $2.72 \pm 0.07$ & $0.57 \pm 0.05$ & $0.89 \pm 0.19$ & 6.5 & 0.8 & 1 \\
\hline HD $283642 \ldots \ldots$ & $2.01 \pm 0.03$ & $0.63 \pm 0.01$ & $1.12+0.08$ & 30.8 & 3.3 & 2 \\
\hline HD $283643 \ldots \ldots$ & $1.35 \pm 0.03$ & $0.64 \pm 0.02$ & $1.01 \pm 0.12$ & 24.6 & 4.9 & 2 \\
\hline HD $283701 \ldots \ldots$. & $3.18 \pm 0.04$ & $0.60 \pm 0.02$ & $0.89 \pm 0.07$ & 33.0 & 1.3 & 1 \\
\hline HD $283725 \ldots \ldots$. & $4.84 \pm 0.07$ & $0.50 \pm 0.02$ & $0.79 \pm 0.06$ & 66.4 & 1.2 & 1 \\
\hline HD $283757 \ldots \ldots$. & $2.92 \pm 0.02$ & $0.62 \pm 0.01$ & $1.05 \pm 0.06$ & 40.2 & 2.0 & 2 \\
\hline HD $283800 \ldots \ldots$ & $3.95 \pm 0.11$ & $0.53 \pm 0.02$ & $0.86 \pm 0.14$ & 27.0 & 0.9 & 1 \\
\hline HD $283809 \ldots \ldots$ & $6.70 \pm 0.10$ & $0.59 \pm 0.02$ & $0.97 \pm 0.10$ & 52.4 & 4.1 & 1 \\
\hline HD $283812 \ldots \ldots$ & $6.30 \pm 0.09$ & $0.55 \pm 0.02$ & $0.96 \pm 0.07$ & 31.8 & 1.3 & 1 \\
\hline HD $283815 \ldots \ldots$ & $2.86 \pm 0.04$ & $0.61 \pm 0.01$ & $1.11 \pm 0.06$ & 45.8 & 1.3 & 2 \\
\hline HD $283855 \ldots \ldots$ & $5.13 \pm 0.09$ & $0.51 \pm 0.01$ & $0.91 \pm 0.07$ & 46.0 & 0.6 & 1 \\
\hline HD $283877 \ldots \ldots$ & $1.65 \pm 0.04$ & $0.65 \pm 0.01$ & $1.01 \pm 0.11$ & 27.4 & 1.5 & 2 \\
\hline HD $283879 \ldots \ldots$ & $4.24 \pm 0.04$ & $0.65 \pm 0.01$ & $1.22 \pm 0.04$ & 29.6 & 2.0 & 2 \\
\hline Elias $3 \ldots \ldots \ldots \ldots$ & $2.48+0.03$ & $0.69 \pm 0.02$ & $1.03+0.07$ & 108.3 & 0.8 & 2 \\
\hline Elias $29 \ldots \ldots \ldots$ & $4.51 \pm 0.08$ & $0.59 \pm 0.02$ & $1.12 \pm 0.10$ & 30.7 & 0.9 & 2 \\
\hline Elias $30 \ldots \ldots \ldots$ & $5.49 \pm 0.05$ & $0.64 \pm 0.01$ & $1.24 \pm 0.05$ & 33.9 & 1.5 & 2 \\
\hline
\end{tabular}

REFERENCES. - (1) Whittet et al. 1992. (2) This paper, Table 1.

Survey (2MASS): the first and second releases (1999 May and $2000 \mathrm{March}$ ) together cover almost all of the Taurus region. 2MASS provides a database of stellar photometry in passbands centered at $1.25 \mu \mathrm{m}(J), 1.65 \mu \mathrm{m}(H)$ and $2.17 \mu \mathrm{m}$ $\left(K_{S}\right)^{6}$ In general, any differences between 2MASS filter characteristics and those of other near infrared photometric systems in common use (e.g., Bessell \& Brett 1988) should

\footnotetext{
${ }^{6}$ See the 2MASS World Wide Web site at http:// pegasus.phast.umass.edu for full details.
}

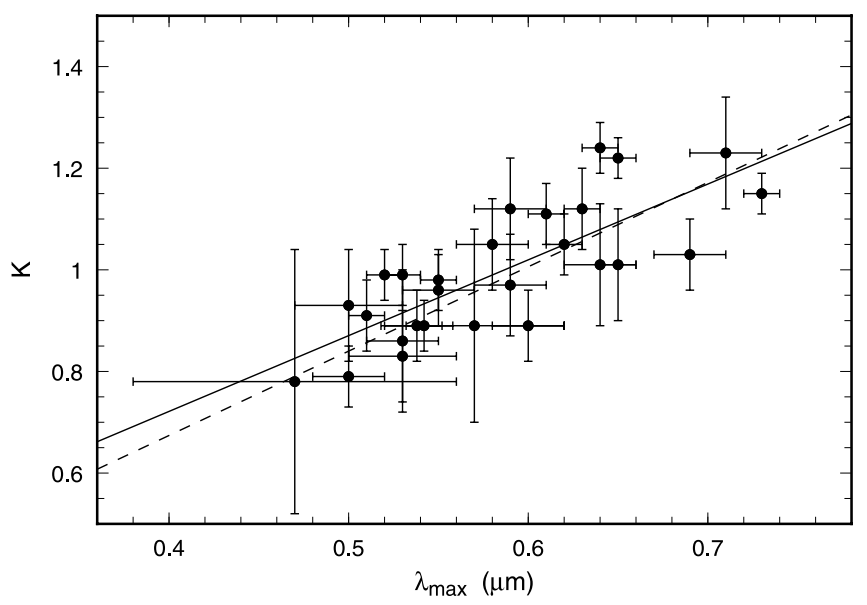

FIG. 1.-Plot of the Serkowski "width" parameter $K$ against $\lambda_{\max }$, using data from Table 2 . The solid line is a linear least-squares fit to the points. The dashed line is the linear relationship adopted by Whittet et al. (1992) as representative of the interstellar medium over a range of environments. be very small. The internal calibration of 2MASS photometry is thought to be extremely precise. Relationships between 2MASS and other photometric systems are currently under investigation and not yet firmly established. An important concern in this paper is to match observed and intrinsic colors.

We compared 2MASS photometry with previous $J H K$ photometry, as available, for stars in the Taurus region: the data are listed in Table 3. 2MASS magnitudes are presented as they appear in the database without rounding. Nominal errors in individual 2MASS $J, H$, and $K$ magnitudes are typically \pm 0.03 , and in no case do they exceed \pm 0.05 . In addition to results from previous literature, the comparison photometry in Table 3 includes previously unpublished UKIRT observations for 12 stars. The latter were obtained on the night of 1988 December 15, using the solid nitrogencooled infrared spectrophotometer UKT6 and standard $J H K$ passbands. Observational uncertainties in these magnitudes are typically \pm 0.02 or less. Standard stars for zeropoint calibration were selected from Elias et al. (1982), and results are on the CIT photometric system.

Figures 2 and 3 show correlation plots (2MASS vs. literature) for the two color indices most relevant to this study, i.e., $V-K$ and $J-K$. The $V$ magnitudes used and their references are listed in Tables 4 and 5. Infrared data of different authors are distinguished by plotting symbol; all literature values have been transformed to the CIT system as necessary. ${ }^{7}$ Also shown in Figures 2 and 3 are linear least-squares fits to the complete data set in each case. The

\footnotetext{
${ }^{7}$ Infrared photometry from Vrba \& Rydgren (1985) and Straižys et al. (1985) is on the Kitt Peak system, which is similar to the Johnson system.
} 
TABLE 3

COMPARISON OF 2MASS AND LITERATURE $J H K$ PHOTOMETRY

\begin{tabular}{|c|c|c|c|c|c|c|c|}
\hline \multirow[b]{2}{*}{ STAR } & \multicolumn{3}{|c|}{ 2MASS } & \multicolumn{3}{|c|}{ LITERATURE $^{\mathrm{a}}$} & \multirow[b]{2}{*}{ REFERENCES $^{\mathrm{b}}$} \\
\hline & $J$ & $H$ & $K$ & $J$ & $H$ & $K$ & \\
\hline HD $27405 \ldots \ldots \ldots$ & 7.290 & 7.294 & 7.275 & 7.32 & $\ldots$ & 7.31 & 1 \\
\hline HD 27778 A....... & 5.900 & 5.892 & 5.902 & 5.96 & $\ldots$ & 5.94 & 1 \\
\hline HD $28149 \ldots \ldots .$. & 5.642 & 5.717 & 5.731 & 5.70 & $\ldots$ & 5.76 & 1 \\
\hline HD $28170 \ldots \ldots . .$. & 7.695 & 7.576 & 7.463 & 7.76 & 7.57 & 7.49 & 2 \\
\hline HD $28975 \ldots \ldots \ldots$ & 7.415 & 7.210 & 7.097 & 7.47 & 7.24 & 7.13 & 3 \\
\hline HD $28991 \ldots \ldots . .$. & 7.058 & 6.994 & 6.845 & 7.08 & $\ldots$ & 6.89 & 1 \\
\hline HD $29333 \ldots \ldots .$. & 7.140 & 6.959 & 6.824 & 7.16 & 6.96 & 6.85 & 2 \\
\hline \multirow[t]{2}{*}{ HD $29647 \ldots \ldots \ldots$} & 5.932 & 5.578 & 5.359 & 5.98 & $\ldots$ & 5.41 & 1 \\
\hline & & & & 5.92 & 5.57 & 5.36 & 3 \\
\hline HD $29835 \ldots \ldots \ldots$ & 5.754 & $\ldots$ & 4.906 & 5.78 & 5.11 & 4.96 & 2 \\
\hline HD $30168 \ldots \ldots . .$. & 6.882 & 6.865 & 6.773 & 6.94 & 6.85 & 6.81 & 2 \\
\hline HD $30675 \ldots \ldots . .$. & 6.707 & 6.687 & 6.641 & 6.79 & 6.71 & 6.69 & 2 \\
\hline HD $279652 \ldots \ldots$ & 8.941 & 8.840 & 8.785 & 8.92 & 8.80 & 8.74 & 2 \\
\hline HD $279658 \ldots \ldots$ & 8.590 & 8.458 & 8.458 & 8.63 & 8.49 & 8.44 & 2 \\
\hline HD $283367 \ldots \ldots$. & 8.954 & 8.780 & 8.630 & 8.99 & 8.77 & 8.67 & 3 \\
\hline HD $283550 \ldots \ldots$ & 7.204 & 7.057 & 6.955 & 7.22 & $\ldots$ & 6.97 & 1 \\
\hline \multirow[t]{2}{*}{ HD $283637 \ldots \ldots$} & 9.522 & 9.285 & 9.190 & 9.50 & 9.28 & 9.16 & 2 \\
\hline & & & & 9.57 & 9.32 & 9.18 & 3 \\
\hline HD $283701 \ldots \ldots$ & 7.792 & 7.561 & 7.435 & 7.81 & 7.60 & 7.48 & 2 \\
\hline HD $283725 \ldots \ldots$ & 7.912 & 7.599 & 7.425 & 7.93 & 7.57 & 7.46 & 2 \\
\hline HD $283740 \ldots \ldots$ & 9.547 & 9.454 & 9.405 & 9.44 & $\ldots$ & 9.32 & 1 \\
\hline HD $283800 \ldots \ldots$ & 8.845 & 8.751 & 8.698 & 8.83 & 8.73 & 8.68 & 2 \\
\hline HD $283809 \ldots \ldots$ & 6.967 & 6.455 & 6.162 & 7.00 & 6.44 & 6.14 & 4 \\
\hline HD $283855 \ldots \ldots$ & 9.517 & 9.349 & 9.243 & 9.47 & 9.28 & 9.20 & 2 \\
\hline VR c .............. & 7.841 & 7.585 & 7.364 & 7.94 & 7.60 & 7.42 & 3 \\
\hline VR d .............. & 10.188 & 9.960 & 9.837 & 10.25 & 10.00 & 9.85 & 3 \\
\hline VR e .............. & 9.606 & 9.351 & 9.187 & 9.61 & 9.33 & 9.17 & 3 \\
\hline Elias $4 \ldots \ldots \ldots \ldots$ & 7.577 & 6.858 & 6.673 & 7.57 & 6.90 & 6.73 & 5 \\
\hline
\end{tabular}

${ }^{a}$ CIT photometric system unless otherwise indicated.

b References: 1, 2, 3, and 6 are as in Table 4; (12) Arce \& Goodman 1999.

correlation coefficients are 0.999 and 0.994 for $V-K$ and $J-K$, respectively. The fits result in the following transformations:

$$
\begin{aligned}
(V-K)_{\mathrm{CIT}}= & (1.000 \pm 0.005)(V-K)_{2 \mathrm{MASS}} \\
& -(0.013 \pm 0.005)
\end{aligned}
$$

and

$(J-K)_{\mathrm{CIT}}=(0.94 \pm 0.01)(J-K)_{2 \mathrm{MASS}}+(0.02 \pm 0.01)$.

Our transformation for $J-K$ (eq. [4]) is consistent with the results of an independent study by J. Carpenter (2000, private communication). These equations were used to homogenize the photometry (effectively tying 2MASS to CIT) prior to calculation of color excesses.

\subsection{Color Excesses and Extinction Results}

A catalog of spectral classifications, color excesses, visual extinctions and $R_{V}$ values for our polarimetry program stars is presented in Table 4, together with bibliographic information. Corresponding data for additional reddened stars (lacking polarimetry) appears in Table 5. Intrinsic $B-V$ colors used to calculate $E_{B-V}$ are taken from Schmidt-Kaler (1982). $E_{V-K}$ and $E_{J-K}$ were calculated using infrared photometry from 2MASS, averaged with data from other sources (Table 3) where available, all data being tied to the CIT photometric system $(\S 2.2)$. Intrinsic $V-K$ and $J-K$ colors were taken from Bessell \& Brett
(1988) and Glass (1997), transformed to the CIT system using equations given by these authors. ${ }^{8}$ Luminosity class $\mathrm{V}$ was assumed for stars lacking two-dimensional classifications.

Determination of $R_{V}$ and $A_{V}$ for individual stars is based on the assumption

$$
R_{V}=\left[\frac{E_{V-\lambda}}{E_{B-V}}\right]_{\lambda \rightarrow \infty}
$$

(e.g., Whittet 1992). This extrapolation is expected to hold unless there is a substantial population of very large grains that give neutral (wavelength-independent) extinction, a possibility that seems inconsistent with observational evidence and abundance constraints (Whittet 1992). In practice, $\lambda$ in equation (5) is set to the longest-wavelength infrared passband for which photometry is available. An approximation consistent with theoretical models,

$$
R_{V} \approx 1.1 \frac{E_{V-K}}{E_{B-V}}
$$

(Whittet \& van Breda 1978), is adopted here, in common with many other investigations of interstellar extinction. The error in $R_{V}$ for a given star depends primarily on the uncertainty of its spectral type and on the degree of extinc-

\footnotetext{
${ }^{8}$ Note that Glass (1997) includes early (O-B8) spectral types not covered by the Bessell \& Brett (1988) compilation.
} 


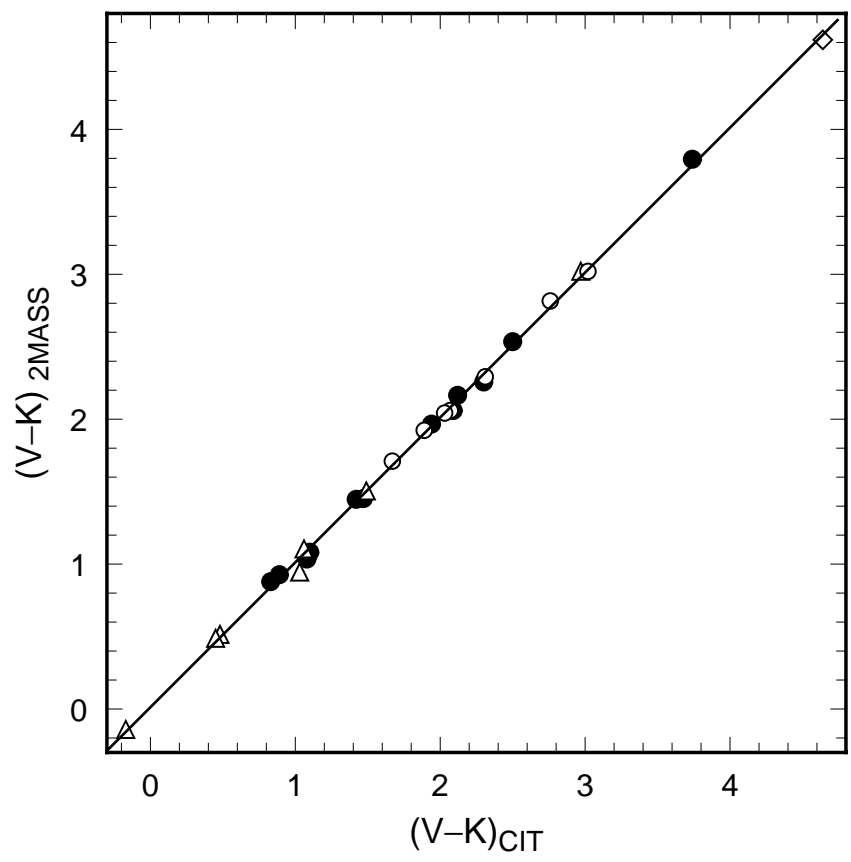

Fig. 2.-Correlation of $V-K$ colors based on 2MASS and literature infrared photometry for stars listed in Table 3 . Literature values are from this paper (solid circles), Vrba \& Rydgren (1985; open circles); Kenyon et al. (1994; upright triangles); Straižys et al. (1985; diamonds) and Elias (1978a; inverted triangles). All literature values have been transformed to the CIT system. The unweighted linear least-squares fit to all data points is shown.

tion; \pm 0.15 is a representative value. It follows from equation (6) that

$$
A_{V} \approx 1.1 E_{V-K} .
$$

This relation was used to calculate $A_{V}$ for most individual stars in our study.

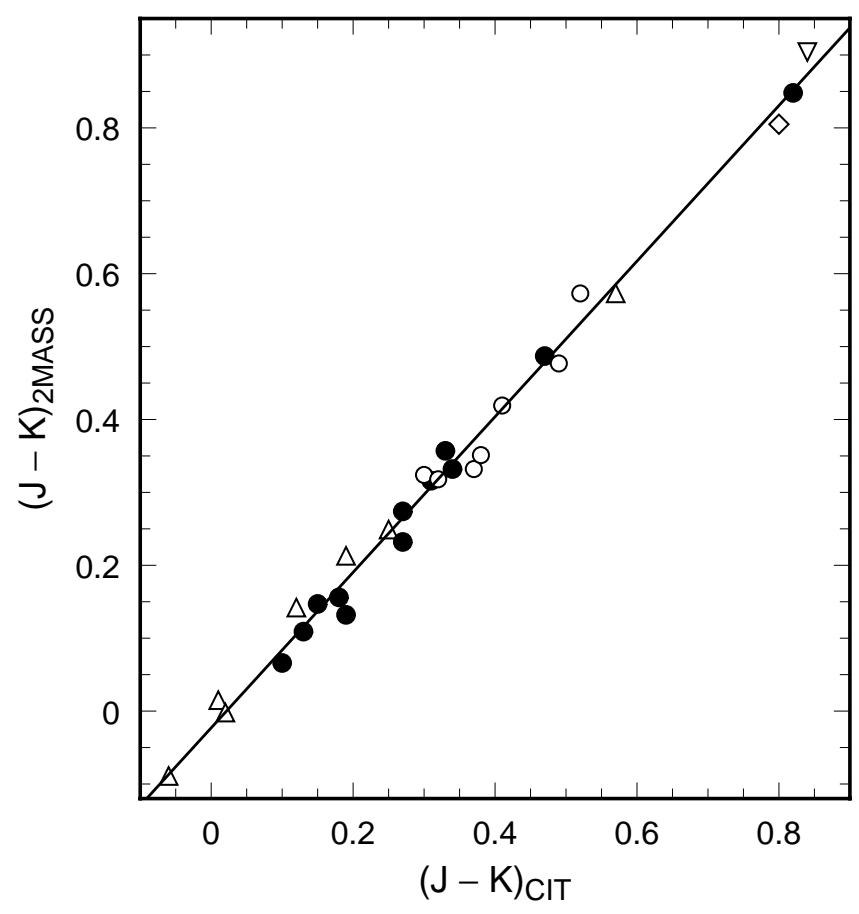

FIg. 3.-Same as Fig. 2, but for $J-K$

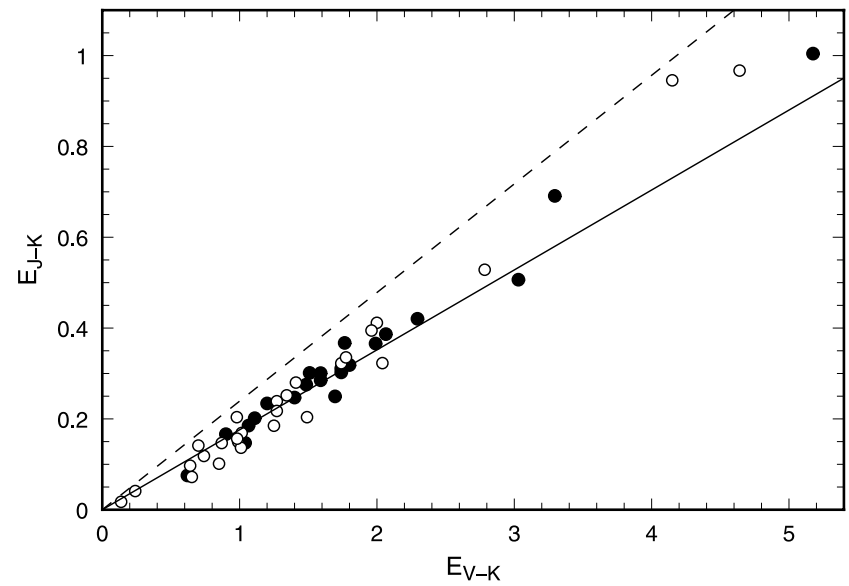

FIG. 4.-Plot of $E_{J-K}$ vs. $E_{V-K}$. Filled circles denote stars from the polarimetry sample (Table 4 ) and open circles denote other reddened stars (Table 5). The solid line is the linear least-squares fit through the origin to all points with $E_{J-K}<0.6$, with slope equivalent to $r=A_{V} / E_{J-K}=6.2$. The dashed line is the extinction law $(r=4.6)$ adopted by Elias (1978a).

Estimates of $A_{V}$ for reddened stars that lack visual photometry (of which there are four in Table 4) can be made by extrapolation from an infrared color excess such as $E_{J-K}$ :

$$
A_{V}=r E_{J-K},
$$

where $r$ is the ratio of total to selective near-infrared extinction. The value of $r$ depends on the form of the interstellar extinction curve over the relevant spectral range. Elias (1978a) adopted $r=4.6$ to calculate $A_{V}$ toward field stars detected in his $2 \mu \mathrm{m}$ survey of the TDC - a value actually determined from the extinction curve of the star S- 1 in the $\rho$ Ophiuchi dark cloud (Elias 1978b). However, Smith, Sellgren, \& Brooke (1993) propose $r \approx 5.4$ in the TDC, a value closer to that determined for diffuse (low-density) regions of the ISM $(r \approx 5.9$; Torres-Dodgen, Tapia, \& Carrol 1991; He et al. 1995 and references therein).

The plot of $E_{J-K}$ versus $E_{V-K}$ for our sample (Fig. 4) provides constraints on the value of $r$ appropriate to dust in the TDC. This correlation is predicted to be a straight line through the origin of slope $1.1 /\langle r\rangle$ for dust with uniform optical properties. The best linear fit through the origin to all data points with $E_{J-K}<0.6$ (solid line) yields $\langle r\rangle=6.2 \pm 0.4$, a value indistinguishable from that for the diffuse-ISM. Note, however, that some of the most highly reddened stars in Figure 4 tend to have lower $r$, and thus plot closer to the Elias extinction law $(r=4.6$; dashed line $)$. We calculated $A_{V}$ for stars lacking $B$ and $V$ photometry in Table 4 using equation (8) with $r=6\left(E_{J-K}<0.6\right)$ and $r=5.3\left(E_{J-K}>0.6\right)$. The latter value represents a compromise between the Elias and diffuse-ISM extinction laws; it will tend to overestimate the extinction toward stars with the highest color excesses (e.g., Elias 13) if $r$ decreases systematically with increasing $A_{V}$ in the inner regions of the cloud.

\section{ANALYSIS}

\subsection{The Threshold Extinction}

As discussed in $\S 1$, the threshold extinction $\left(A_{\mathrm{th}}\right)$ for ice absorption is a key parameter in our characterization of the diffuse/dense cloud interface. $A_{\text {th }}$ is determined from the correlation of $A_{V}$ with the optical depth $\left(\tau_{3.0}\right)$ of the $3 \mu \mathrm{m}$ ice 
TABLE 4

Spectral Classifications and Extinction Data for Polarimetry Program Stars

\begin{tabular}{|c|c|c|c|c|c|c|c|c|}
\hline Star & Spectral Type & $V$ & $E_{B-V}$ & $E_{V-K}$ & $E_{J-K}$ & $A_{V}{ }^{\mathrm{a}}$ & $R_{V}$ & References \\
\hline HD $28170 \ldots \ldots \ldots$ & A3 V & 8.91 & 0.44 & 1.20 & 0.23 & 1.32 & 3.00 & 1 \\
\hline HD $28225 \ldots \ldots \ldots$ & A3 III & 7.78 & 0.41 & 1.11 & 0.20 & 1.22 & 2.98 & 1,2 \\
\hline HD $28975 \ldots \ldots \ldots$ & A4 III & 9.02 & 0.60 & 1.59 & 0.29 & 1.75 & 2.92 & $1,2,3$ \\
\hline HD $29333 \ldots \ldots \ldots$ & A2 V & 8.79 & 0.62 & 1.80 & 0.32 & 1.98 & 3.19 & 2,4 \\
\hline HD $29647 \ldots \ldots \ldots$ & B6-7 IVp & 8.38 & 1.00 & 3.30 & 0.69 & 3.63 & 3.63 & 5 \\
\hline HD $29835 \ldots \ldots \ldots$ & K2 III & 8.70 & 0.38 & 1.04 & 0.15 & 1.14 & 3.01 & 2,6 \\
\hline HD $30168 \ldots \ldots \ldots$ & B8 V & 7.70 & 0.45 & 1.07 & 0.19 & 1.18 & 2.62 & 1,6 \\
\hline HD $30675 \ldots \ldots \ldots$ & B3 V & 7.52 & 0.51 & 1.40 & 0.25 & 1.54 & 3.02 & 1,2 \\
\hline HD $279652 \ldots . .$. & $\mathrm{A} 2 \mathrm{~V}$ & 9.82 & 0.31 & 0.90 & 0.17 & 0.99 & 3.19 & 7 \\
\hline HD $279658 \ldots \ldots \ldots$ & B7 V & 9.91 & 0.67 & 1.70 & 0.25 & 1.87 & 2.79 & 7 \\
\hline HD $283367 \ldots \ldots .$. & B9 V & 10.34 & 0.65 & 1.77 & 0.37 & 1.95 & 3.00 & 2,3 \\
\hline HD $283637 \ldots \ldots$. & $\mathrm{A} 0 \mathrm{~V}$ & 11.25 & 0.78 & 2.07 & 0.39 & 2.28 & 2.92 & 2,3 \\
\hline HD $283642 \ldots \ldots \ldots$ & A3 V & 10.60 & 0.68 & 1.99 & 0.37 & 2.19 & 3.22 & 6 \\
\hline HD $283643 \ldots \ldots .$. & $\mathrm{A} 2 \mathrm{~V}$ & 10.93 & 0.56 & 1.51 & 0.30 & 1.66 & 2.97 & 6,8 \\
\hline HD $283701 \ldots \ldots$. & B8 III & 9.60 & 0.89 & 2.30 & 0.42 & 2.53 & 2.84 & 2 \\
\hline HD $283725 \ldots \ldots .$. & F5 III & 9.96 & 0.61 & 1.59 & 0.30 & 1.75 & 2.87 & 1 \\
\hline HD $283757^{b} \ldots \ldots$ & A5 V & 10.93 & 0.55 & $\ldots$ & $\ldots$ & 1.7: & $\ldots$ & 6 \\
\hline HD $283800 \ldots \ldots$. & B5 V & 9.78 & 0.53 & 1.49 & 0.28 & 1.64 & 3.09 & 6 \\
\hline HD $283809 \ldots \ldots$. & B3 V & 10.78 & 1.61 & 5.18 & 1.00 & 5.70 & 3.54 & 9 \\
\hline HD $283812 \ldots \ldots$. & $\mathrm{A} 1 \mathrm{~V}$ & 9.48 & 0.71 & 1.74 & 0.31 & 1.91 & 2.70 & $1,2,6$ \\
\hline HD $283815 \ldots \ldots .$. & A5 V & 9.75 & 0.57 & 1.74 & 0.30 & 1.91 & 3.35 & 6 \\
\hline HD $283855 \ldots \ldots .$. & A2 & $\ldots$ & $\ldots$ & $\ldots$ & 0.28 & 1.7: & $\ldots$ & 2 \\
\hline HD $283877 \ldots \ldots$. & F5 V & 9.95 & 0.23 & 0.65 & 0.08 & 0.72 & 3.11 & 8 \\
\hline HD $283879 \ldots \ldots$. & B5 $\mathrm{V}^{\mathrm{c}}$ & 11.08 & 1.18 & 3.03 & 0.51 & 3.33 & 2.82 & 8,10 \\
\hline Elias $3 \ldots \ldots \ldots \ldots$ & K2 III & $\ldots$ & $\ldots$ & $\ldots$ & 1.88 & 10.0: & $\ldots$ & 11 \\
\hline Elias $29 \ldots \ldots \ldots \ldots$ & G9 III & $\ldots$ & $\ldots$ & $\ldots$ & 0.80 & 4.2: & $\ldots$ & 11 \\
\hline Elias $30 \ldots \ldots \ldots \ldots$ & K0 III & $\ldots$ & $\ldots$ & $\ldots$ & 0.92 & 4.9: & $\ldots$ & 11 \\
\hline
\end{tabular}

${ }^{a}$ Visual extinction is calculated from $A_{V}=1.1 E_{V-K}$ for all stars with both $U B V$ and $J H K$ photometry available. In other cases (values followed by colons): $A_{V}=3.0 E_{B-V}$ (stars lacking $J H K$ photometry); $A_{V}=$ $r E_{J-K}$ (stars lacking $U B V$ photometry), with $r=6\left(E_{J-K}<0.6\right)$ and $r=5.3\left(E_{J-K}>0.6\right)$.

${ }^{b}$ HD 283757 is the only star in our sample lacking infrared photometry: by chance it falls in a narrow strip of sky missed by 2 MASS scans released to date.

${ }^{\mathrm{c}}$ No reliable spectral classification of HD 283879 exists in the literature: it is currently listed as an M star in Simbad, and as Ap, F5, or F8 by Slutskii, Stal'bovskii, \& Shevchenko (1980). Our adopted spectral type of B5 V is based on careful analysis of $U B V$ and $J H K$ colors. These colors are clearly incompatible with a classification as late as $\mathrm{M}$ for any value of reddening. The locus of the star in the $U-B, B-V$ plane is consistent with either $\sim \mathrm{B} 5$ or $\sim \mathrm{F} 5$; its locus in the $J-H, H-K$ plane is consistent with a reddened B-type star and inconsistent with an F-type star.

REFERENCES.-References for spectral types and $U B V$ photometry are as follows: (1) Kenyon et al. 1994; (2) Simbad database; (3) Vrba \& Rydgren 1985; (4) Oja 1987; (5) Crutcher 1985; (6) Straižys \& Meištas 1980; (7) Ungerer et al. 1985; (8) Slutskii et al. 1980; (9) Straižys et al. 1985; (10) This paper, see footnote c; (11) Elias 1978a.

absorption feature. Recent observations reported by Murakawa, Tamura, \& Nagata (2000) have greatly increased the number of stars with $\tau_{3.0}$ and $A_{V}$ values available, and a reassessment is therefore timely. Figure 5 plots $\tau_{3.0}$ versus $A_{V}$ for all available lines of sight with $A_{V}>2$ and $\tau_{3.0} \geq$ 0.05 , using data from Whittet et al. (1988), Smith et al. (1993) and Murakawa et al. (2000). The Smith et al. sample (nine stars) is a subset of the Whittet et al. sample (12 stars), and these have been combined (filled circles), taking average values of $\tau_{3.0}$ for common stars; $A_{V}$ is taken from our current work as available (Table 4: HD 29647, HD 283809, Elias 3) or otherwise estimated from equation (8) with $r=5.3$. The Murakawa et al. results (open circles) are presented as they appear in Table 2 of that paper, except that we have substituted our optically-determined $A_{V}$ values for their values (based on $A_{V}=12 E_{H-K}$ ) for HD 29647 and HD 283809.

The distribution of points in Figure 5 shows no evidence for a systematic difference between the data sets that might arise, for example through different methods of evaluating $A_{V}$. The unweighted linear least squares fit to all 36 points, shown in Figure 5, is represented by

$$
\tau_{3.0}=q\left(A_{V}-A_{\mathrm{th}}\right)
$$

with $q=0.072 \pm 0.002$ and $A_{\mathrm{th}}=3.2 \pm 0.1$. This result for the threshold extinction is in good agreement with the value of 3.3 originally proposed by Whittet et al. (1988) for a much smaller sample. There does not appear to be strong evidence in Figure 5 for intrinsic variations proposed by Murakawa et al. (2000) in the distribution of ice with $A_{V}$ in the dark-cloud environment. We therefore adopt $A_{\mathrm{th}}=3.2$.

\subsection{The Ratio of Total to Selective Extinction}

$R_{V}$-values measured in dense clouds generally range from the diffuse-ISM average of about 3.0 up to $\sim 5.5$ in some regions of active star formation such as M42 (e.g., Whittet 1992 and references therein). Systematic increases in $R_{V}$ with total extinction are predicted if stars with high $A_{V}$ sample dense regions of the cloud, as the rate of grain growth $(\S 1)$ should increase with density. Such behavior has been reported previously for other dark clouds, most 
TABLE 5

Extinction Data for Additional Reddened Stars

\begin{tabular}{|c|c|c|c|c|c|c|c|c|}
\hline Star & Spectral Type & $V$ & $E_{B-V}$ & $E_{V-K}$ & $E_{J-K}$ & $A_{V}^{\mathrm{a}}$ & $R_{V}$ & References \\
\hline HD $27405 \ldots \ldots \ldots$ & B8 V & 7.79 & 0.25 & 0.65 & 0.07 & 0.72 & 2.86 & 1 \\
\hline HD 27778 A....... & B3 V & 6.39 & 0.38 & 1.02 & 0.17 & 1.12 & 2.95 & 1 \\
\hline HD $27778 B \ldots \ldots$ & $\mathrm{A} 0 \mathrm{~V}$ & 8.19 & 0.32 & 0.85 & 0.10 & 0.94 & 2.92 & 1 \\
\hline HD $27877 \ldots \ldots \ldots$ & B9 $\mathrm{V}$ & 7.36 & 0.42 & 1.27 & 0.24 & 1.40 & 3.33 & 12,6 \\
\hline HD $27923 \ldots \ldots \ldots$ & B9 V & 8.13 & 0.27 & 0.64 & 0.10 & 0.70 & 2.61 & 1 \\
\hline HD $28149 \ldots \ldots \ldots$ & B5 V & 5.59 & 0.11 & 0.24 & 0.04 & 0.26 & 2.4: & 1 \\
\hline HD $28272 \ldots \ldots \ldots$ & A6 V & 9.02 & 0.39 & 0.98 & 0.20 & 1.08 & 2.76 & 1 \\
\hline HD $28482 \ldots \ldots \ldots$ & B7 V & 7.13 & 0.56 & 1.41 & 0.28 & 1.55 & 2.77 & 1 \\
\hline HD $28991 \ldots \ldots \ldots$ & F3 V & 7.95 & 0.05 & 0.14 & 0.02 & 0.15 & 3.1: & 1 \\
\hline HD $30067 \ldots \ldots \ldots$ & $\mathrm{A} 2 \mathrm{~V}$ & 8.97 & 0.36 & 0.87 & 0.15 & 0.96 & 2.66 & 1 \\
\hline HD $31252 \ldots \ldots \ldots$ & $\mathrm{A} 0 \mathrm{~V}$ & 8.47 & 0.32 & 0.99 & 0.15 & 1.09 & 3.40 & 1,2 \\
\hline HD $283550 \ldots \ldots$ & $\mathrm{A} 2 \mathrm{~V}$ & 8.46 & 0.50 & 1.34 & 0.25 & 1.47 & 2.95 & 1 \\
\hline HD $283553 \ldots \ldots$ & B6 V & 9.07 & 0.41 & 1.01 & 0.14 & 1.11 & 2.71 & 1,2 \\
\hline HD $283592 \ldots \ldots$. & A4 V & 9.86 & 0.26 & 0.74 & 0.12 & 0.81 & 3.13 & 1 \\
\hline HD $283740 \ldots \ldots$ & A0 V & 10.35 & 0.36 & 0.98 & 0.16 & 1.08 & 2.99 & 1 \\
\hline HD $283920 \ldots \ldots$. & $\mathrm{A} 0 \mathrm{~V}$ & 9.12 & 0.61 & 1.49 & 0.20 & 1.64 & 2.69 & 1 \\
\hline HD $283929 \ldots \ldots$ & A5 V & 9.37 & 0.45 & 1.27 & 0.19 & 1.38 & 3.06 & $1,2,6$ \\
\hline HD $283932 \ldots \ldots$. & B7 V & 9.92 & 0.75 & 2.04 & 0.32 & 2.24 & 2.99 & 1 \\
\hline HD $283939 \ldots \ldots$ & $\mathrm{A} 2 \mathrm{~V}$ & 10.29 & 0.41 & 1.27 & 0.22 & 1.40 & 3.41 & 1,2 \\
\hline HD $284012 \ldots \ldots$. & B6 V & 8.29 & 0.62 & 1.74 & 0.32 & 1.91 & 3.09 & 1 \\
\hline HD $284014 \ldots \ldots$. & B5 V & 9.35 & 0.79 & 2.00 & 0.41 & 2.20 & 2.78 & 1 \\
\hline HD $284287 \ldots \ldots$ & F2 III & 8.90 & 0.25 & 0.70 & 0.14 & 0.77 & 3.08 & 1 \\
\hline VR c............. & A0 & 10.18 & 1.00 & 2.79 & 0.53 & 3.07 & 3.07 & 3 \\
\hline VR d ............. & A1 & 11.88 & 0.66 & 1.96 & 0.40 & 2.16 & 3.27 & 3 \\
\hline VR e $\ldots . . . \ldots \ldots . .$. & A7 & 11.48 & 0.68 & 1.78 & 0.34 & 1.96 & 2.88 & 3 \\
\hline $091085 \ldots \ldots \ldots \ldots$ & G0 & 16.96 & 1.54 & 4.64 & 0.97 & 5.10 & 3.31 & 12 \\
\hline $141134 \ldots \ldots \ldots \ldots$ & A5 & 14.29 & 1.19 & 4.15 & 0.95 & 4.57 & 3.84 & 12 \\
\hline
\end{tabular}

${ }^{\text {a }}$ Calculated from $A_{V}=1.1 E_{V-K}$.

REFERENCES.- (1) Kenyon et al. 1994; (2) Simbad database; (3) Vrba \& Rydgren 1985; (4) Oja 1987; (5) Crutcher 1985; (6) Straižys \& Meištas 1980; (7) Ungerer et al. 1985; (8) Slutskii et al. 1980; (9) Straižys et al. 1985; (10) This paper, see footnote c; (11) Elias 1978a; (12) Arce \& Goodman 1999.

notably $\rho$ Ophiuchi (Carrasco et al. 1973; Vrba, Croyne, \& Tapia 1993), where $R_{V} \sim 4.0-4.5$ is typical of the densest regions. As the highest value found for any star in our sample is a mere 3.8 , it is evident that the TDC is dominated by dust broadly similar to that in the diffuse ISM, at least

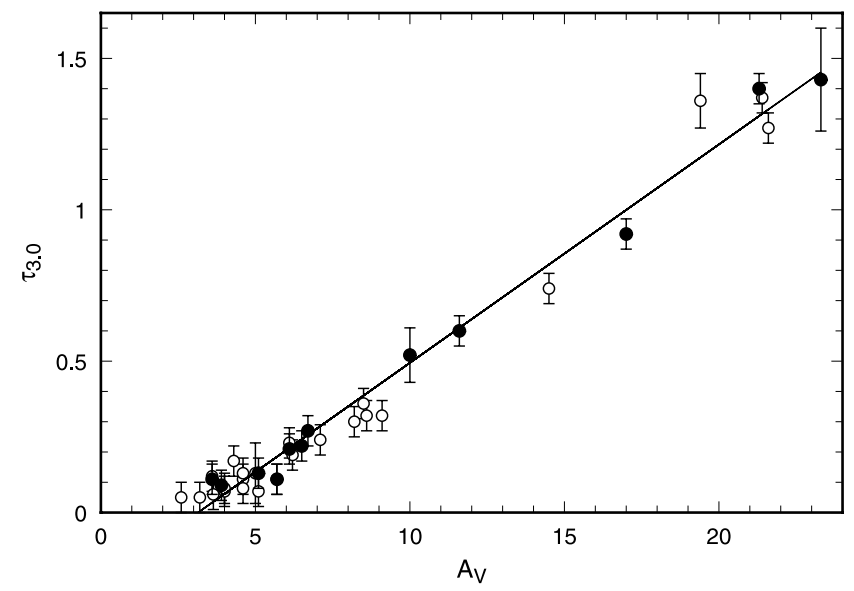

FIG. 5.-Plot of ice optical depth $\tau_{3.0}$ vs. extinction $A_{V}$ for the Taurus dark cloud. Filled circles: 12 stars from the sample of Whittet et al. (1988), with $\tau_{3.0}$ values averaged from Whittet et al. (1988) and Smith et al. (1993); error bars represent scatter between the two sets of observations where this exceeds 0.05. Open circles: 24 stars from the sample of Murakawa et al. (2000). Only stars with $A_{V}>2$ and $\tau_{3.0} \geq 0.05$ are included. The line is the unweighted linear least-squares fit to all data points. The intercept on the $A_{V}$ axis yields a threshold extinction of $A_{\mathrm{th}}=3.2 \pm 0.1$. over the extinction range sampled. In this section, we examine in detail the evidence for systematic changes in $R_{V}$.

The frequency distribution of $R_{V}$ in the TDC is plotted as a bar chart in Figure 6. For comparison, we show the distribution for a typical sample of the diffuse-ISM (toward highly reddened OB stars; He et al. 1995), represented by a gaussian profile centered at $R_{V}=3.05$ with $\sigma=0.3$ (dashed

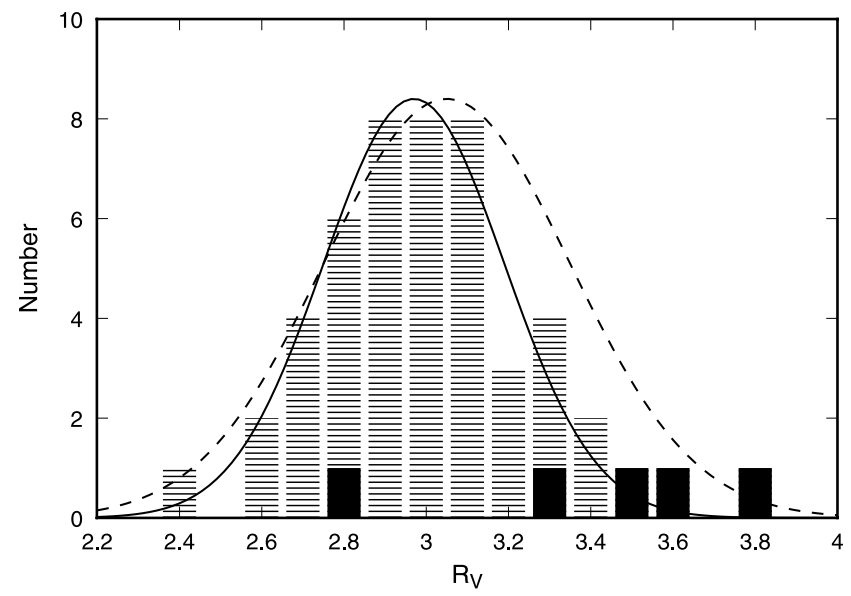

FIG. 6.-Bar chart of $R_{V}$ values for stars in the Taurus cloud, obtained by plotting numbers of stars in bins of width 0.1 . Stars with $A_{V}>3.2$ (i.e., above the ice threshold) are shown as solid bars. The continuous curve is a Gaussian distribution (centered at $R_{V}=2.97$ with $\sigma=0.22$ ) fitted by eye to the Taurus data. The dashed curve (centered at $R_{V}=3.05$ with $\sigma=0.3$ ) represents the diffuse-ISM data of $\mathrm{He}$ et al. (1995). 
curve). Although this curve fits reasonably well on the low$R_{V}$ side of the peak, the TDC data are better represented by a narrower profile $(\sigma=0.22)$ centered at 2.97 (continuous curve). Stars with $3.1<R_{V}<3.5$ seem to be somewhat under-represented in the TDC compared with the diffuse ISM. Note, however, that four out of five stars with $A_{V}>$ $A_{\text {th }}$ have above-average $R_{V}$ values.

The $E_{B-V}$ versus $E_{V-K}$ diagram, plotted in Figure 7, provides the clearest evidence for a real change in the optical properties of the dust as the extinction increases. The plot should resemble a straight line through the origin of slope $1.1 /\left\langle R_{V}\right\rangle$ (by analogy with Fig. 4) if $R_{V}$ is the same everywhere and scatter is due only to random observational error. The distribution of points in Figure 7 suggests that this prediction does, indeed, hold true for stars with $E_{V-K}<2.9$ (equivalent to $A_{V}<3.2$, i.e., below the ice threshold). The least-squares fit through the origin to these stars (solid line) yields $\left\langle R_{V}\right\rangle=2.97 \pm 0.15$ (consistent with the peak in Fig. 6). At greater $E_{V-K}$, the slope appears to change systematically in the direction of higher $R_{V}$. Note that the four stars with highest $E_{V-K}$ values in Figure 7 $\left(E_{B-V}\right.$ vs. $\left.E_{V-K}\right)$ are displaced (relative to the correlation line) in the opposite sense compared with their displacements in Figure $4\left(E_{J-K}\right.$ vs. $\left.E_{V-K}\right)$, i.e., increasing $R_{V}$ appears to be accompanied by decreasing $r$.

The $R_{V}$ values for individual stars are plotted against $A_{V}$ in Figure 8. Points with $A_{V}<3.2$ are distributed about $R_{V}=2.97$, represented by the horizontal line in Figure 8 . Scatter might arise in part from real star-to-star differences in addition to observational error, but no systematic trends are apparent for the low- $A_{V}$ stars. The vertical line represents the ice threshold extinction. Two stars with $A_{V} \approx$ $A_{\text {th }}$ have normal $R_{V}$, whilst four stars with $A_{V}>A_{\text {th }}$ have above-average $R_{V}$. A logical interpretation of the data is that only stars with extinctions appreciably above the ice threshold sample regions dense enough for systematic grain growth leading to enhanced $R_{V}$.

\subsection{The Wavelength of Maximum Polarization}

Previous work has shown that $\lambda_{\max }$ is generally quite well correlated with $R_{V}$, consistent with the expectation that each of these parameters varies in proportion to mean grain

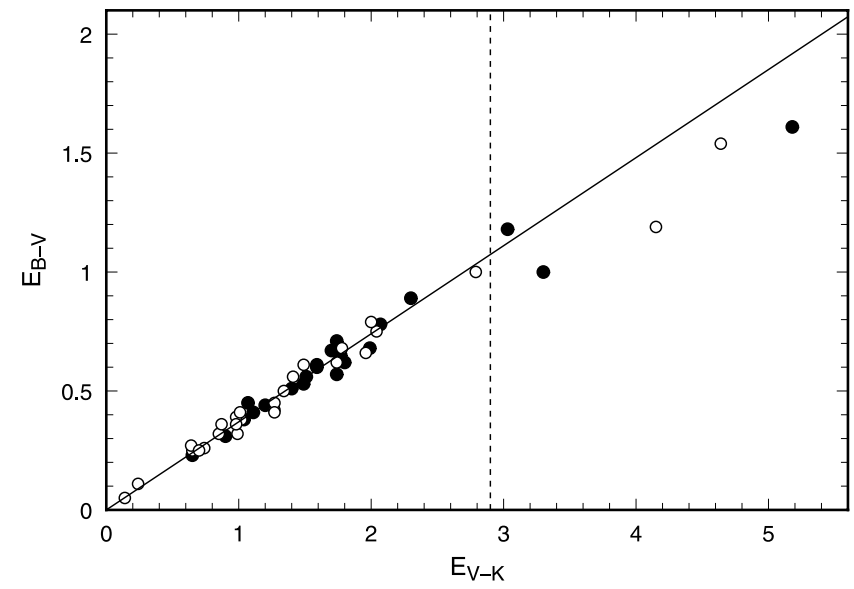

FIG. 7.-Plot of $E_{B-V}$ vs. $E_{V-K}$. Plotting symbols have the same meaning as in Fig. 4. The vertical dashed line represents the ice threshold $\left(E_{V-K}=A_{\mathrm{th}} / 1.1=2.9\right)$. The solid line is the linear least-squares fit through the origin for points below the ice threshold, with slope equivalent to $\left\langle R_{V}\right\rangle=2.97 \pm 0.15$.

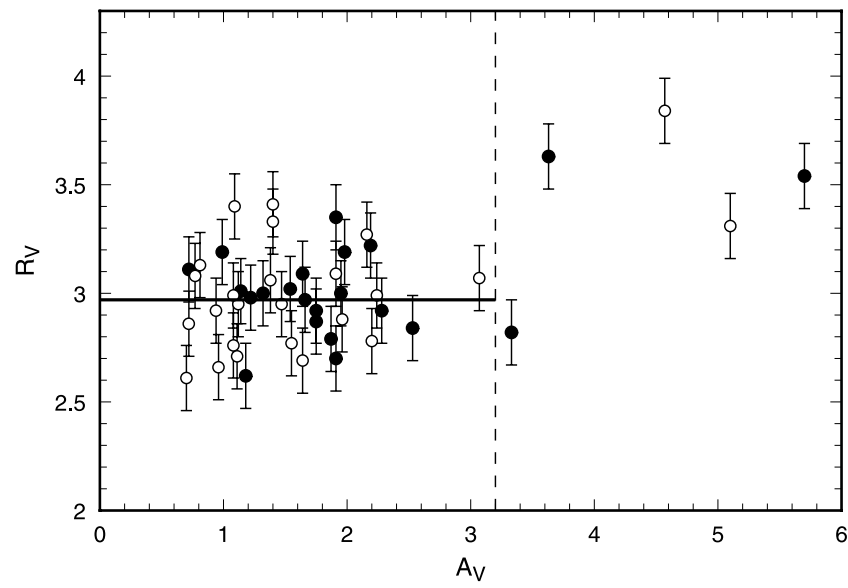

Fig. 8.-Plot of $R_{V}$ vs. $A_{V}$. Symbols have the same meaning as in Fig. 4. The vertical dashed line represents the ice threshold extinction, $A_{\mathrm{th}}=3.2$. The horizontal line represents the mean value $\left\langle R_{V}\right\rangle=2.97$ for $A_{V}<A_{\mathrm{th}}$, deduced from Fig. 7.

size (Serkowski 1973; Serkowski et al. 1975; Whittet \& van Breda 1978; Clayton \& Mathis 1988; Vrba et al. 1993; Whittet et al. 1994; Larson, Whittet, \& Hough 1996). Whittet \& van Breda (1978) found that

$$
R_{V}=(5.6 \pm 0.3) \lambda_{\max }
$$

adequately describes this correlation for a variety of interstellar environments from diffuse to dark clouds, although the scatter is often greater than can be explained purely in terms of observational error (see Clayton \& Mathis 1988). The plot of $R_{V}$ versus $\lambda_{\max }$ for the TDC is shown in Figure 9 (HD 279652 is omitted from this and subsequent plots because of the large uncertainty in $\left.\lambda_{\max }\right)$. Remarkably, Figure 9 shows little correlation. There is a clustering of points around the ISM average $\left(R_{V} \approx 3.1, \lambda_{\max } \approx 0.55 \mu \mathrm{m}\right)$, and the highest- $R_{V}$ star (HD 29647) also has the highest $\lambda_{\max }$; but otherwise there is no clear trend of increasing $R_{V}$ with $\lambda_{\max }$ (compare the predicted correlation from eq. [10]). Apparently, $\lambda_{\max }$ can have a wide range of values $(\sim 0.50$ $0.71 \mu \mathrm{m})$ toward stars with $R_{V}$-values that do not deviate

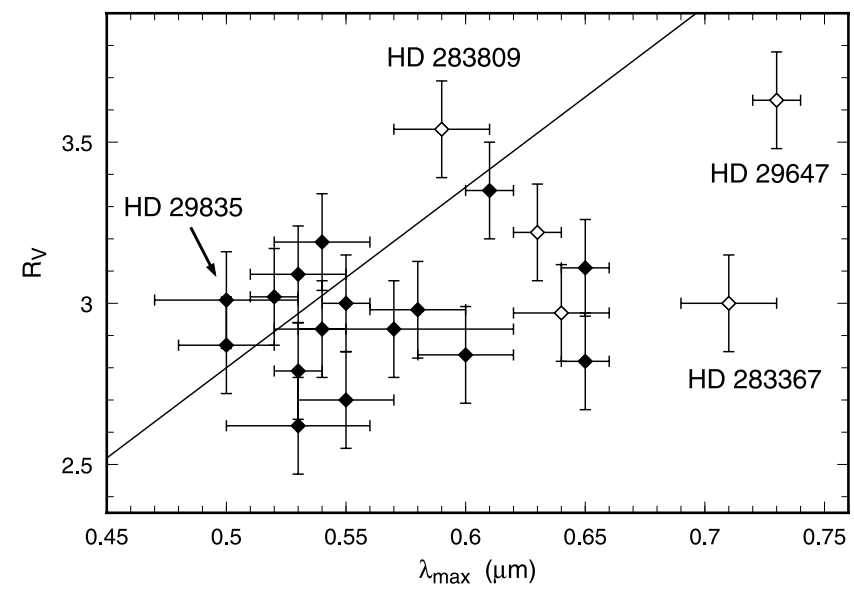

FIG. 9.-Plot of $R_{V}$ vs. $\lambda_{\max }$ for stars in the polarimetry sample. Data are from Tables 4 and 2, respectively. Open symbols represent five stars with significant position-angle rotation with respect to wavelength (§ 2.1), filled symbols those with essentially constant position angle. The diagonal line is the mean relationship $R_{V}=5.6 \lambda_{\max }$ deduced by Whittet \& van Breda (1978). 
significantly from the mean. Compare, for example, HD 283367 and HD 283725 in Figure 9: both have $R_{V}$ values close to 3.0 but their $\lambda_{\max }$ values differ by $\sim 40 \%$.

We hypothesize that the unexpectedly poor degree of correlation between $\lambda_{\max }$ and $R_{V}$ is caused by size-dependent variations in grain alignment. In the past, $\lambda_{\max }$ has been widely used as a proxy for mean grain size, but $\lambda_{\max }$ is actually capable of two kinds of behavior, which we term "size-dependent" and "alignment-dependent" behavior. Previous discussions have emphasized size-dependent behavior. However, several studies have shown that the polarizing efficiency of interstellar dust, as measured by the ratio $p / A$ at some wavelength, declines systematically with total extinction, as one probes progressively denser environments within a dark cloud (e.g., Goodman et al. 1992; Whittet et al. 1994; Gerakines, Whittet, \& Lazarian 1995; Arce et al. 1998). That this holds true for the present sample is illustrated in Figure 10, which plots $p_{\max } / A_{V}$ against $A_{V}$. The curve represents a fit of the form adopted by Gerakines et al. (1995). Note that for $A_{V}<A_{\mathrm{th}}, p_{\max } / A_{V}$ has a wide range of values, but for $A_{V}>A_{\mathrm{th}}, p_{\max } / A_{V}$ is systematically low ( $<1.5 \% \mathrm{mag}^{-1}$; for comparison, the general interstellar mean is $\sim 3 \% \mathrm{mag}^{-1}$ ). A number of factors may affect polarizing efficiency in a given line of sight. Stars in our sample with significant $\theta$-rotation have low $p_{\max } / A_{V}$, suggesting depolarization resulting from superposition of distinct cloud components (Messinger, Whittet, \& Roberge 1997). However, a systematic decline in polarization efficiency with increasing $A_{V}$ is evident even if stars with $\theta$ rotation are ignored. The most probable cause is a corresponding reduction in the efficiency of the alignment mechanism (see Gerakines et al. 1995 for discussion). Although the alignment mechanism is not fully understood (Roberge 1996), the degree of alignment achieved in a given environment is predicted to be a strong function of grain size for either superparamagnetic or suprathermal alignment (Mathis 1986; Lazarian \& Draine 1997), i.e., in marginal conditions, it is the small grains that lose their alignment first. As the small grains polarize most efficiently at shorter wavelengths, $\lambda_{\max }$ will shift toward longer wavelengths as their orientations become randomized. This change will be independent of $R_{V}$ if the size distribution of

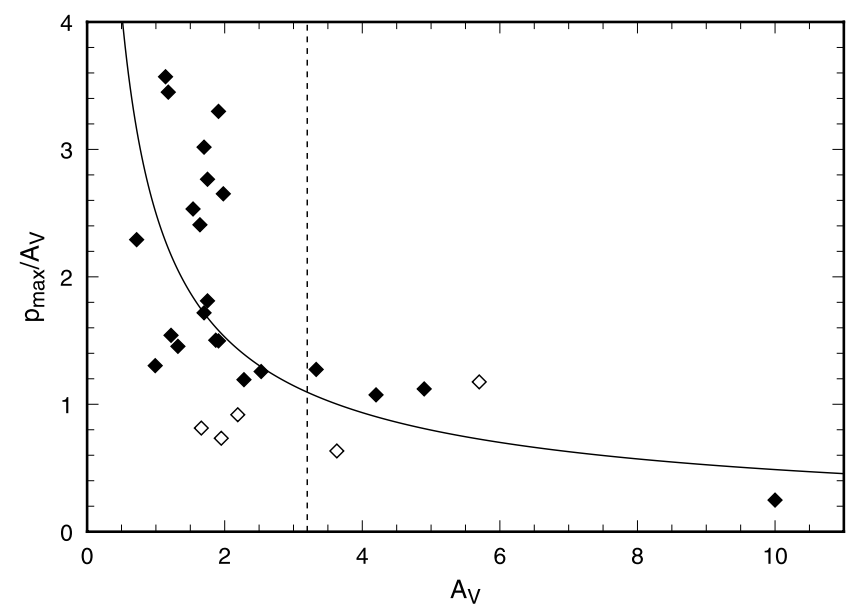

FIG. 10.-Plot of polarizing efficiency $\left(p_{\max } / A_{V}\right)$ vs. $A_{V}$. Symbols have the same meaning as in Fig. 9. The dashed vertical line represents the ice threshold extinction, $A_{\mathrm{th}}=3.2$. The curve is a fit of the form $y=a x^{b}$ (Gerakines et al. 1995) with $a=2.5$ and $b=-0.71$. all grains (summed over aligned and unaligned components) remains the same.

Figures 11 and 12 compare correlations of $\lambda_{\max }$ with extinction $A_{V}$ and polarizing efficiency $p_{\max } / A_{V}$, respectively. The scatter in $\lambda_{\max }$ above the value predicted by $R_{V}$ (eq. [10]) at low $A_{V}$ is evident in Figure 11 (compare Fig. 8). Whereas Figure 11 shows a weak positive correlation, Figure 12 shows a rather stronger negative correlation (the correlation coefficients are 0.45 and -0.76 , respectively). To test whether this situation is peculiar to the TDC, we examined corresponding plots for the Chamaeleon I dark cloud using data from Whittet et al. (1994), and found it to exhibit similar behavior: the correlation coefficients are $0.62\left(\lambda_{\max }\right.$ vs. $\left.A_{V}\right)$ and $-0.67\left(\lambda_{\max }\right.$ vs. $\left.p_{\max } / A_{V}\right)$ for Chamaeleon I. These results are consistent with the hypothesis of high $\lambda_{\max }$ arising from reduced alignment efficiency.

Does $\lambda_{\max }$ carry any useful information on the integrated grain size distribution in the TDC that can be used to study grain growth? Our results clearly indicate that it cannot be

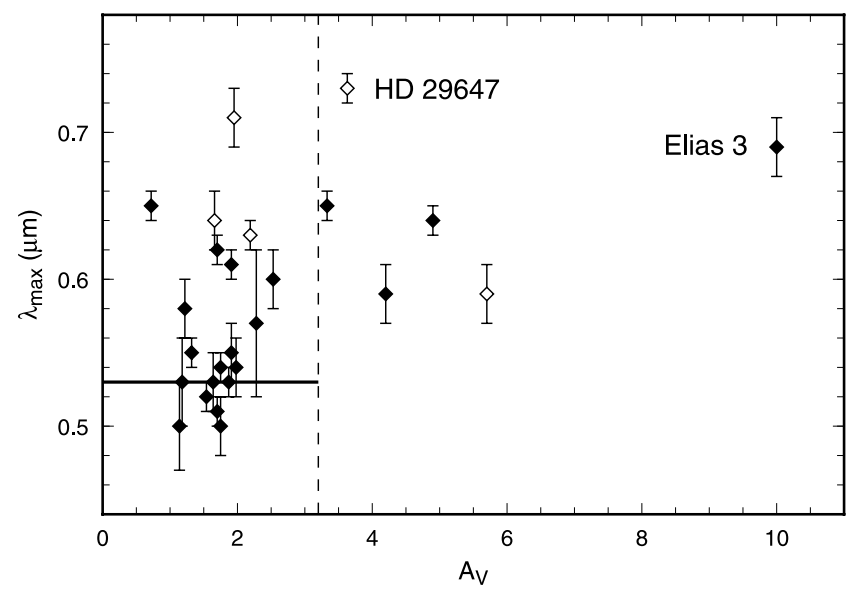

FIG. 11.- Plot of $\lambda_{\max }$ vs. $A_{V}$. Symbols have the same meaning as in Fig. 9. The dashed vertical line represents the ice threshold extinction, $A_{\mathrm{th}}=3.2$. The horizontal line represents the value of $\lambda_{\max }$ equivalent to the mean value of $R_{V}$ using equation (10), i.e., $\left\langle\lambda_{\max }\right\rangle=2.97 / 5.6=0.53 \mu \mathrm{m}$.

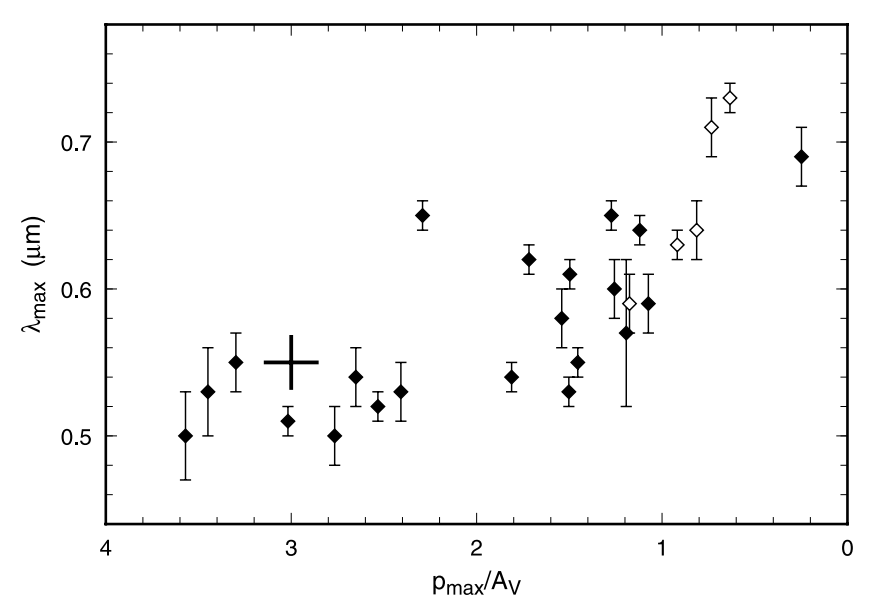

FIG. 12.-Plot of $\lambda_{\max }$ vs. $p_{\max } / A_{V}$. The cross indicates the locus of the general diffuse-ISM average, other symbols have the same meaning as in Fig. 9. The $p_{\max } / A_{V}$ axis is reversed to facilitate comparison with other plots, i.e., extinction increases from left to right. 


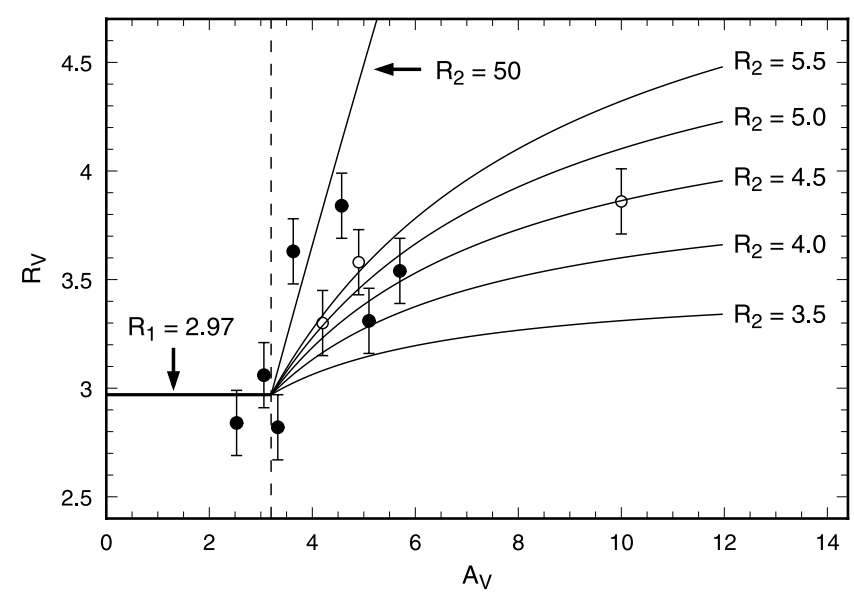

Fig. 13.-Dependence of $R_{V}$ on $A_{V}$, according to the simple model described in $\S 4$, for several values of the parameter $R_{2}$. The dashed vertical line represents the ice threshold extinction, $A_{\mathrm{th}}=3.2$, and the horizontal line represents the mean value of $R_{V}\left(R_{1}=2.97\right)$ at $A_{V}<A_{\mathrm{tb}}$. For clarity, only stars with $A_{V}>2.5$ are plotted (filled circles: $R_{V}$ determined photometrically; open circles: $R_{V}=5.6 \lambda_{\max }$ assumed).

relied upon at extinctions $A_{V}<A_{\mathrm{th}}$, where as many as $30 \%-40 \%$ of the stars have $\lambda_{\max }$ values significantly greater than predicted from their photometric $R_{V}$ values. However, alignment-dependent behavior of $\lambda_{\max }$ appears to saturate quickly at higher $A_{V}$. Toward HD 29647 and HD 283809, enhanced $\lambda_{\max }$ is accompanied by enhanced $R_{V}$ on approximately the expected scale (Fig. 9), indicating real changes in grain properties. The $R_{V}$ value toward Elias $3\left(A_{V} \approx 10\right)$ is unknown, but it is notable that its $\lambda_{\max }$ value, although high, is no greater than that of HD 29647 (see Fig. 11). An explanation for this might be that the alignment of grains deep within the cloud is so inefficient that dust at low-to-intermediate $A_{V}$ dominates the polarization curve; in the case of Elias 3, this does seem probable given its low degree of polarization. However, mantled grains within the cloud must align to some extent, as they produce significant excess polarization corresponding to the $3 \mu \mathrm{m}$ ice absorption feature (Hough et al. 1988; Kobayashi et al. 1999). With these caveats in mind, we tentatively assume that the $R_{V} \approx$ $5.6 \lambda_{\max }$ relationship gives a meaningful estimate of $R_{V}$ for stars with $A_{V}>A_{\text {th }}$.

\section{A SIMPLE MODEL FOR THE DEPENDENCE OF $R_{V}$ ON $A_{V}$}

A realistic model for the general structure of the TDC appears to be a screen of diffuse material in which clumps of denser material are embedded (Messinger et al. 1997; Arce \& Goodman 1999; Murakawa et al. 2000). The observed dependence of polarization position-angle on wavelength toward HD 29647 and HD 283809 can be understood if both grain properties (size and/or composition) and the orientation of the magnetic field in the clump (TMC-1) differs from that in the screen (Messinger et al. 1997). The existence of an ice threshold extinction is consistent with this scenario: ice absorption is seen only in lines of sight that intercept a clump. Stars behind clumps have (at least) two components to their extinctions, one arising in the pervasive screen, and one in the clump (or clumps). We deduce the dependence of $R_{V}$ on $A_{V}$, as predicted by this simple model, on the assumption that each component has uniform optical properties. This assumption is undoubtedly simplistic, but it proves to be in reasonable accord with available observational constraints.

The ratio of total to selective extinction of a star behind a clump may be written

$$
R_{V}=\frac{A_{V}}{A_{1} / R_{1}+A_{2} / R_{2}},
$$

where $A_{V}=A_{1}+A_{2}$ and subscripts 1 and 2 represent the diffuse screen and the clump, respectively. Note that "screen extinction" $A_{1}$ presumably arises in two parts, one in front of the clump and one behind, but this need not concern us here as the two parts add linearly. For $A_{V}>A_{\mathrm{th}}$, we have $A_{1}=A_{\mathrm{th}}$ and $A_{2}=A_{V}-A_{\mathrm{th}}$, thus

$$
R_{V}=\frac{A_{V}}{A_{\mathrm{th}} / R_{1}+\left(A_{V}-A_{\mathrm{th}}\right) / R_{2}} .
$$

Empirical values for $R_{1}(2.97)$ and $A_{\mathrm{th}}(3.2)$ have been established by our work (\$ 3.1-3.2). Equation (12) thus allows the dependence of $R_{V}$ on $A_{V}\left(>A_{\mathrm{th}}\right)$ to be plotted for an assumed value of $R_{2}$. For stars not behind clumps, $R_{V}=$ $R_{1}=2.97\left(0<A_{V}<A_{\mathrm{th}}\right)$ is assumed.

Several values of $R_{2}$ were used in equation (12) to generate representative curves, plotted in Figure 13. Also shown for comparison is the distribution of empirical points for high-extinction stars $\left(A_{V}>2.5\right)$ in our sample. Figure 13 shows that the trend of increasing $R_{V}$ predicted by the simple screen/clump model with $R_{2} \sim 4.5$ is generally consistent with the observed behavior of $R_{V}$ (or $\lambda_{\max }$ ) with respect to $A_{V}$ around or above the ice threshold. Two exceptions (HD 29647 and Arce-Goodman object 141134) require very much higher values of $R_{2}(\sim 50)$ to give a sufficiently steep rise in $R_{V}$ with $A_{V}$ above $A_{\mathrm{th}}$. It may well be the case that these lines of sight are not described adequately by our model. For example, the screen might be unusually thin, allowing a greater contribution to the total extinction from clump grains. A more intriguing possibility is that these objects might, in fact, be embedded within the cloud, in which case the effective value of $A_{\mathrm{th}}$ in equation (12) should be reduced (e.g., halved if equal columns of screen material lie in front of and behind the star); the curves in Figure 13 should then be displaced to the left, accordingly. However, at least in the case of HD 29647, this scenario seems inconsistent with available evidence, such as the lack of a reflection nebula (see Crutcher 1985 for discussion).

\section{MANTLE GROWTH VERSUS COAGULATION: CLUES FROM UV EXTINCTION}

Are the changes in mean grain size implied by increasing $R_{V}$ toward stars with the highest dust-columns (Fig. 13) the result of mantle growth, coagulation, or a combination of both? It is certainly suggestive that $R_{V}$ begins to change at $A_{V} \approx A_{\mathrm{th}}$, the threshold extinction for the presence of $\mathrm{H}_{2} \mathrm{O}$ ice mantles. Thus, some fraction of the grains in the high- $R_{V}$ lines of sight are evidently mantled. Extinction curves at ultraviolet (UV) wavelengths provide a valuable means of discrimination: the level of UV continuum extinction in normalized extinction curves should decline with increasing $R_{V}$ if small grains are systematically incorporated into larger ones by coagulation (Cardelli et al. 1989; Mathis 1996), whilst the width of the $2175 \AA$ bump is sensitive to mantling (Mathis 1994). Regrettably, suitable UV spectra 


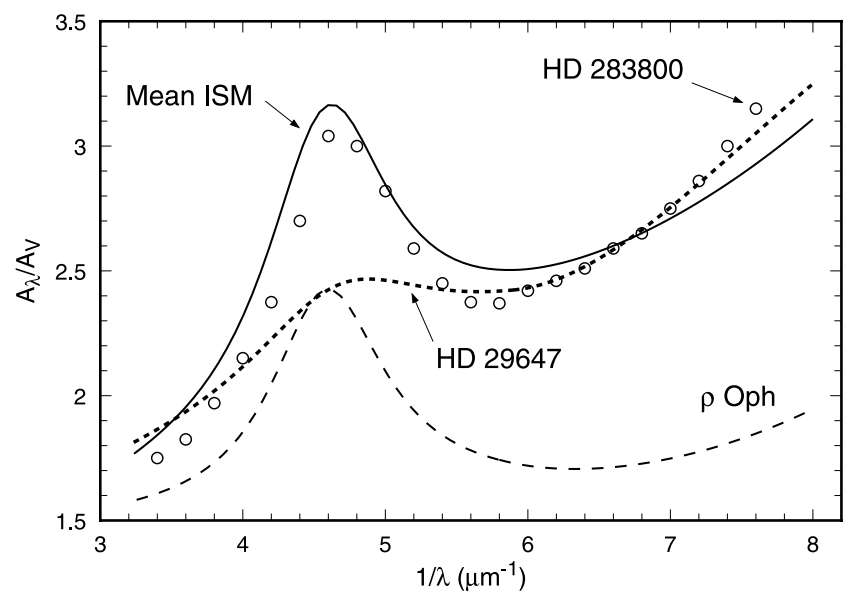

FIG. 14.- Ultraviolet extinction curves of Taurus stars HD 29647 and HD 283800, compared with those of HD 147933/4 ( $\rho$ Oph) and the mean interstellar medium. The HD 283800 curve is from Adamson (1999, private communication). The HD 29647, $\rho$ Oph and mean ISM curves were calculated from empirical formulae (Fitzpatrick \& Massa 1988), using parameters from Cardelli \& Savage (1988) and Fitzpatrick \& Massa (1988); this gives a close representation of the original data. Color excess ratios $E_{\lambda-V} / E_{B-V}$ were converted to normalized extinction $A_{\lambda} / A_{V}$ using the appropriate $R_{V}$ value in each case.

are available for only two lines of sight through the TDC: the extinction curve of HD 283800 was determined from spectra obtained with the International Ultraviolet Explorer satellite $(I U E)$ by A. J. Adamson (1999, private communication), and that of HD 29647 (also determined from IUE data) was presented and discussed in detail by Cardelli \& Savage (1988). ${ }^{9}$ Thus, we have one example of a star with $A_{V}<A_{\mathrm{th}}$ and one with $A_{V}>A_{\mathrm{th}}$, illustrated in Figure 14. Also shown are the mean diffuse-ISM and $\rho$ Oph extinction curves (from Fitzpatrick \& Massa 1988).

The extinction curve of HD 283800 (Fig. 14) is quite similar to the diffuse-ISM average curve, consistent with its normal $R_{V}$ value $(\sim 3.1$; Table 4$)$, providing further illustration of the generally unremarkable properties of dust at $A_{V}<A_{\mathrm{th}}$ in the TDC. In contrast, the extinction curves of $\rho$ Oph and HD 29647 show substantial (and differing) departures from the norm:

$\rho O p h$. - The UV continuum extinction is relatively weak, whilst the $2175 \AA$ Aump has near-normal strength. The difference between the $\rho$ Oph and mean-ISM curves is characteristic of the $R_{V}$-dependent behavior $\left(R_{V} \approx 4.1\right.$ vs. 3.0$)$ described by Cardelli et al. (1989). Coagulation is evidently the process that governs the grain size distribution toward $\rho$ Oph (Jura 1980; Vrba et al. 1993), whereas ice-mantle growth appears to be unimportant at extinctions $A_{V}<10$ (Harris, Woolf, \& Rieke 1978; Tanaka et al. 1990). Coagulation naturally leads to reduced UV continuum extinction.

HD 29647.- The $2175 \AA$ bump is much broader and weaker than in the mean ISM, whilst the UV continuum extinction is relatively strong. The breadth of the bump may

\footnotetext{
${ }^{9}$ Note that HD 29647 has a peculiar intrinsic spectrum with anomalously strong $\mathrm{Hg}$ and Mn lines (e.g., Crutcher 1985). This increases the problem of finding a suitable comparison spectrum to determine the UV extinction curve by the standard pair method, as discussed in detail by Cardelli \& Savage (1988). These authors conclude that the UV extinction curve is truly anomalous and not significantly influenced by spectral mismatching.
}

be attributed to mantling of small graphite grains, ${ }^{10}$ and the strength of the UV continuum extinction suggests that coagulation is relatively unimportant. These findings strengthen the argument that mantle growth is the dominant process in the TDC.

\section{SUMMARY AND OVERVIEW}

The results of this paper provide insight into the development of grain properties with extinction as we observe stars through progressively more opaque (and denser) regions of the TDC. At low $A_{V}(0-3 \mathrm{mag})$, the dust is almost indistinguishable, in terms of its optical properties, from that in the general diffuse ISM. However, its alignment properties are already changing: this change is characterized by a systematic decline in polarizing efficiency with increasing $A_{V}$, accompanied by enhanced $\lambda_{\max }$ attributed to selective failure of the smaller grains to retain their alignment. For $A_{V}>3$, real changes in optical properties of the grains occur, characterized by $R_{V}$ values in the range 3.3-3.8. If the observed extinction toward each star includes a "normal" component as well as a "dense-clump" component, $R_{V}$ may approach $\sim 4.5$ or more for dust within the clumps themselves. The available evidence favors mantle growth over coagulation as the dominant process affecting the optical properties of the dust in the TDC. If changes in $R_{V}$ are not a direct consequence of mantle growth, then they must occur under closely similar physical conditions. Dust in Taurus appears to be in a different evolutionary state compared with other nearby dark clouds, such as $\rho \mathrm{Oph}$, in which coagulation is the dominant process.

Despite our efforts to find information for highly reddened stars, our sample is heavily weighted toward stars with extinctions below $A_{\mathrm{th}}$, which generally have low-tonormal $R_{V}$. It will be important in future work to determine reliable photometric $R_{V}$ values for stars with higher extinctions $\left(A_{V} \gg A_{\mathrm{th}}\right)$, to better constrain the development of grain growth with physical conditions in the cloud. This will require accurate spectral classification and $B V$ photometry for stars with $V \sim 16$. Another important goal for future studies will be to determine whether the UV extinction curves of stars with $A_{V}>A_{\mathrm{th}}$ in the TDC show anomalous behavior similar to that seen so far only in HD 29647. HD 283809 is a prime candidate.

This publication makes use of data products from the Two Micron All Sky Survey, which is a joint project of the University of Massachusetts and the Infrared Processing and Analysis Center, funded by the National Aeronautics and Space Administration (NASA) and the National Science Foundation (NSF). Extensive use was also made of the SIMBAD database, operated at CDS, Strasbourg, France. We thank A. J. Adamson for providing unpublished UV extinction data, and for helpful discussions. We also thank J. M. Carpenter for providing information on 2MASS color transformations in advance of publication. D. C. B. W. gratefully acknowledges support from the NASA Astrophysics Data Program (grant NAG 5-3376) and the NSF Galactic Astronomy Program (grant AST 9419690).

\footnotetext{
${ }^{10}$ Note that pure ice mantles cannot produce the observed increase in the width of the $2175 \AA$ A bump toward HD 29647: Mathis (1994) proposes that the required change in optical constants is caused by incorporation of neutral polycyclic aromatic hydrocarbons into the mantles.
} 


\section{REFERENCES}

Arce, H. G., \& Goodman, A. A. 1999, ApJ, 517, 264

Arce, H. G., Goodman, A. A., Bastien, P., Manset, N., \& Sumner, M. 1998, ApJ, 499, L93

Bessell, M. S., \& Brett, J. M. 1988, PASP, 100, 1134

Cardelli, J. A., Clayton G. C. \& Mathis J. S. 1989, ApJ, 345, 245

Cardelli, J. A., \& Savage, B. D. 1988, ApJ, 325, 864

Carrasco, L., Strom, K. M., \& Strom, S. E. 1973, ApJ, 182, 95

Clayton, G. C., \& Mathis J. S. 1988, ApJ, 327, 911

Clayton, G. C., Wolff, M. J., Allen, R. G., \& Lupie, O. L. 1995, ApJ, 445, 947

Crutcher, R. M. 1985, ApJ, 288, 604

Elias, J. H. 1978a, ApJ, 224, 857 1978 b, ApJ, 224, 453

Elias, J. H., Frogel, J. A., Matthews, K., \& Neugebauer, G. 1982, AJ, 87, 1029

Fitzpatrick, E. L., \& Massa, D. 1988, ApJ, 328, 734

Frerking, M. A., Langer, W. D., \& Wilson, R. W. 1982, ApJ, 262, 590

Gerakines, P. A., Whittet, D. C. B., \& Lazarian, A. 1995, ApJ, 455, L171

Glass, I. S. 1997, Mon. Notes Astron. Soc. South Africa, 56, 110

Goodman, A. A., Jones, T. J., Lada, E. A., \& Myers P. C. 1992, ApJ, 399, 108

Harris, D. H., Woolf, N. J., \& Rieke, G. H. 1978, ApJ, 226, 829

He, L., Whittet, D. C. B., Kilkenny, D., \& Spencer Jones, J. H. 1995, ApJS, 101,335

Hough, J. H., et al. 1988, MNRAS, 230, 107

Hough, J. H., Peacock, T., \& Bailey, J. A. 1991, MNRAS, 248, 74

Jones, A. P. \& Williams, D. A. 1984, MNRAS, 209, 955

Jura, M. 1980, ApJ, 235, 63

Kenyon, S. J., Dobrzycka, D., \& Hartmann, L. 1994, AJ, 108, 1872

Kobayashi, N., Nagata, T., Tamura, M., Takeuchi, T., Takami, H., Kobayashi, Y., \& Sato, S. 1999, ApJ, 517, 256

Larson, K. A., Whittet, D. C. B., \& Hough, J. H. 1996, ApJ, 472, 755

Lazarian, A., \& Draine, B. T. 1997, ApJ, 487, 248

Martin, P. G., Clayton, G. C., \& Wolff, M. J. 1999, ApJ, 510, 905

Mathis, J. S. 1986, ApJ, 308, 281 1994, ApJ, 422, 176

Mathis, J. S. 1996, in ASP Conf. Ser. 97, Polarimetry of the Interstellar Medium, ed. W. G. Roberge \& D. C. B. Whittet (ASP: San Francisco), 3 Meištas, E., \& Straižys, V. 1981, Acta Astron., 31, 85

Messinger, D. W., Whittet, D. C. B., \& Roberge, W. G. 1997, ApJ, 487, 314

Moneti, A., Pipher, J. L., Helfer, H. L., McMillan, R. S., \& Perry, M. L.

1984, ApJ, 282, 508
Murakawa, K., Tamura, M., \& Nagata, T. 2000, ApJS, 128, 603

Oja, T. 1987, A\&A, 68, 211

Roberge, W. G. 1996, in ASP Conf. Ser. 97, Polarimetry of the Interstellar Medium, ed. W. G. Roberge \& D. C. B. Whittet (ASP: San Francisco), 401

Schmidt-Kaler, Th. 1982, in Landolt-Börnstein New Series, Vol. 2b, ed. K. Shaifers \& H. H. Voight (Berlin: Springer)

Serkowski, K. 1973, in IAU Symp. 52, Interstellar Dust and Related Topics, ed. J. M. Greenberg \& H. C. van de Hulst (Dordrecht: Kluwer), 145

Serkowski, K., Mathewson, D. S., \& Ford, V. L. 1975, ApJ, 196, 261

Slutskii, V. E., Stal'bovskii, O. I., \& Shevchenko, V. S. 1980, Soviet Astron. Lett., 6, 397

Smith, R. G., Sellgren, K., \& Brooke, T. Y. 1993, MNRAS, 263, 749

Straižys, V., Cernis, K., \& Hayes, D. S. 1985, Ap\&SS, 112, 251

Straižys, V., \& Meištas, E. 1980, Acta Astron., 30, 541

Tanaka, M., Sato, S., Nagata, T., \& Yamamoto, T. 1990, ApJ, 352, 724

Teixeira, T. C., \& Emerson, J. P. 1999, A\&A, 351, 292

Torres-Dodgen, A. V., Tapia, M., \& Carroll, M. 1991, MNRAS, 249, 1

Ungerer, V., Mauron, N., Brillet, J., \& Nguyen-Quang-Rieu. 1985, A\&A, 146,123

Vrba, F. J., Coyne, G. V., \& Tapia, S. 1993, AJ, 105, 1010

Vrba, F. J., \& Rydgren, A. E. 1985, AJ, 90, 1490

Whittet, D. C. B. 1992, Dust in the Galactic Environment (Bristol: IPP)

Whittet, D. C. B., Bode, M. F., Longmore, A. J., Adamson, A. J., McFadzean, A. D., Aitken, D. K., \& Roche, P. F. 1988, MNRAS, 233, 321

Whittet, D. C. B., Bode, M. F., Longmore, A. J., Baines, D. W. T., \& Evans, A. 1983, Nature, 303, 218

Whittet, D. C. B., Gerakines, P. A., Carkner, A. L., Hough, J. H., Martin, P. G., Prusti, T., \& Kilkenny, D. 1994, MNRAS, 268,

Whittet, D. C. B., Martin, P. G., Hough, J. H., Rouse, M. F., Bailey, J. A., \& Axon, D. J. 1992, ApJ, 386, 562

Whittet, D. C. B., \& van Breda, I. G. 1978, A\&A, 66, 57

Whittet, D. C. B., et al. 1996, ApJ, 458, 363

Wilking, B. A., Lebofsky, M. J., Martin, P. G., Rieke, G. H., \& Kemp, J. C. 1980, ApJ, 235, 905

Wilking, B. A., Lebofsky, M. J., \& Rieke, G. H. 1982, AJ, 87, 695

Williams, D. A., Hartquist, T. W., \& Whittet, D. C.B. 1992, MNRAS, 258, 599 